\title{
Reduced description method in the kinetic theory of Brownian motion with active fluctuations
}

\author{
Oleksii Sliusarenko ${ }^{1,2}$ \\ ${ }^{1}$ BCAM-Basque Center for Applied Mathematics, Alameda de Mazarredo 14, \\ E-48009 Bilbao, Basque Country, Spain \\ ${ }^{2}$ National Science Center "Kharkiv Institute of Physics and Technology", \\ Akademichna Str. 1, 61108 Kharkiv, Ukraine \\ E-mail: osliusarenko@bcamath.org
}

Yurii Slyusarenko ${ }^{1,2}$

${ }^{1}$ National Science Center "Kharkiv Institute of Physics and Technology", Akademichna Str. 1, 61108 Kharkiv, Ukraine

${ }^{2}$ Karazin National University, 4 Svobody Sq., 61077 Kharkiv, Ukraine

E-mail: slusarenko@kipt.kharkov.ua 


\begin{abstract}
.
We develop a microscopic approach to the kinetic theory of many-particle systems with dissipative and potential interactions in presence of active fluctuations. The approach is based on a generalization of Bogolyubov-Peletminskii reduced description method applied to the systems of many active particles. It is shown that the microscopic approach developed allows to construct the kinetic theory of twoand three-dimensional systems of active particles in presence of non-linear friction (dissipative interaction) and an external random field with active fluctuations. The kinetic equations for these systems in case of a weak interaction between the particles (both potential and dissipative ones) and low-intensity active fluctuations are obtained. We demonstrate particular cases in which the derived kinetic equations have solutions that match the results known in the literature. In addition, analysis of particular solutions showed that in the case of a friction force linearly dependent on the speed of structural units, the manifestation of self-propelling properties in the process of evolution of an active medium may be due to the local nature of active fluctuations.
\end{abstract}

PACS numbers: 05.20.-y; 05.20.Dd; 05.10.Gg; 05.40.-a; 05.40.Jc; 45.70.-n; $47.70 \mathrm{Nd}$

Keywords: kinetic theory of gases and liquids, fluctuations, stochastic processes, stochastic particle dynamics, active particles, active matter, microscopical approach, reduced description method, Fokker-Planck equation, self-propulsion solutions

Submitted to: J. Phys. A: Math. Gen.

\title{
1. Introduction
}

Active matter is a field in soft matter physics, which studies the properties of aggregates of self-propulsion objects that have the ability to take up energy from the environment, to store it in an internal depot, and to convert internal energy into kinetic energy. Active matter comprises diverse systems spanning from macroscopic (e.g. schools of fish and flocks of birds) to microscopic scales (e.g. migrating cells, motile bacteria and gels formed through the interaction of nanoscale molecular motors with cytoskeletal filaments within cells). Here we refer the reader to the reviews $[1,2,3,4]$ and references therein. In recent years the number of publications devoted to theoretical and experimental studies of the phenomena in the active substances has sharply increased, and the recent achievements have been mirrored in the Special Issues of the European Physical Journal Special Topics and the Journal of Statistical Physics $[5,6,7,8,9]$. The study of nonequilibrium processes in systems of active particles inevitably raises the question of the consistent derivation of the evolution equations for such systems, in particular, the kinetic equations. The derivation of a kinetic theory of active particles is a challenging issue that attracted significant attention over the past decades. Bertin et al. [10, 11, 12] has derived the Boltzmann equation for the self-propulsion point-like particles on a twodimensional plane with the assumption that the modulus of the velocity vector is fixed 
and identical for all the particles, so that only the direction of the vector plays a role in the dynamics. Ihle $[13,14]$ developed an alternative kinetic approach that is based on the Chapman-Kolmogorov equation for the N-particle probability density. The resulting mean-field kinetic equation has been studied analytically and numerically, and extended to the so-called topological interactions $[15,16,17]$. Romanczuk et al. [18, 19, 20] derived and explored the mean-field kinetic equation in two spatial dimensions, starting from the Langevin equation with active friction and active fluctuations, supplemented with different forces describing interaction between the particles. In Ref. [21] the authors also pursue the Langevin approach to study collective dynamics in two-dimensional system of active Brownian particles with dissipative interactions.

In this paper we develop a consistent microscopic approach based on the reduced description method of relaxation processes. The reduced description method of Bogolyubov-Peletminskii has a number of notable advantages. In particular, it allows one to formulate approaches for the construction of the kinetic theory of systems of many identical particles thus, in essence, providing a dynamic justification of the statistical mechanics of such systems $[22,23]$. N.N. Bogolyubov suggested a method of reduced description of the evolution of many-particle systems [22], which allowed the construction of a regular procedure for obtaining closed dissipative kinetic equations based on the BBGKY chain of reversible equations for many-particle distribution functions. Fundamentals of reduced description method were formulated in [22] for the classical (non-quantum) systems of many particles. In case of quantum many-particle systems the ideas of the Bogolyubov reduced description method were developed in the works by S.V. Peletminskii, and the main results are presented in [23]. There are also other approaches for the dynamical justification of statistical mechanics, which are different from Bogolyubov's approach, for example, in the works of Prigogine's Brussels school [24], as well as different formulations of Bogolyubov's ideas, see, e.g., $[25,26,27,28]$. In the present paper we use the reduced description method in the form close to the one of Bogolyubov-Peletminskii [29], to construct the kinetic theory of many-particle systems with active fluctuations and non-linear friction. For that purpose we need to generalize the canonical Bogolyubov-Peletminskii approach in order to take into account an external stochastic impact and dissipative interactions.

A generalization of the Bogolyubov reduced description method to the case of dissipative many-particle systems in an external stochastic field was first suggested in [30]. In this paper, the authors proposed a formalism for deriving kinetic equations. As a starting point, a stochastic Liouville equation obtained from Hamilton's equations taking dissipation and stochastic perturbations into account was used. The Liouville equation is then averaged over realizations of the stochastic field by an extension of the FurutsuNovikov formula to the case of a non-Gaussian field. As the result, a generalization of the classical Bogolyubov-Born-Green-Kirkwood-Yvon hierarchy is derived. In order to get a kinetic equation for the one-particle distribution function, the authors use a regular breaking procedure of the BBGKY hierarchy by assuming weak interaction between the particles and weak intensity of the field. Within this approximation they get the 
corresponding Fokker-Planck equation for the system in a non-Gaussian stochastic field. Two particular cases by assuming either Gaussian statistics of external perturbation or homogeneity of the system are discussed. In that approach, however, the stochastic external forces do not depend on the velocity (or momentum) of the particles. In other words, the formalism developed in [30] can be applied to the systems with non-linear friction, as it is the case of the active particle systems, but with passive fluctuations of either Gaussian or non-Gaussian nature.

As mentioned above, we announce this work as such, in which a general microscopic approach based on the first principles of statistical physics to the construction of the kinetic theory of such complex systems as dissipative media with active fluctuations, is proposed. These media are proposed to be considered in the manuscript as systems of many identical particles. The structural units of the system are assumed to be point-like, but capable of reacting to an external stochastic effect, depending on the magnitude and direction of their own velocity. This is their main individual characteristic, indicating that the point particles have the property of the so-called head-tail asymmetry. In particular, the manifestation of the self-propulsion property of the medium itself can be associated with just this simplest characteristic of the asymmetry of structural units. In this paper, we show that in the studied model of the active medium, the self-propulsion property can manifest itself as a collective effect, however, its very manifestation is possible only due to the individual properties of the structural units of the system, the active particles.

Actually, one of the main goals of this work was to demonstrate both the necessity and the very possibility of developing a microscopic approach to building the kinetics of such complex systems with accurate formulations approaching the level of such of mathematical physics. In addition, we formulated the task of developing such a method, which in a certain sense would unite the existing phenomenological approaches (if possible, without contradictions) with their generalization to a number of more complex systems with active fluctuations. First of all, it means the correct inclusion of potential and dissipative interactions between the structural units of the systems under study. In parallel, the aim was to take into account the possibility of influencing the system of spatially correlated noise, depending on the absolute value and the direction of the velocity of its structural unit. At the same time, the formulations should allow for a generalization of the approach to the case of non-Gaussian noise. All stated goals could be achieved only within the framework of a microscopic approach based on the first principles of statistical physics. In this sense, we consider reference works $[22,23,24,25,26,27]$ with a preference for strict approaches and elegant formulations of the monograph [23]. This means that the scheme for constructing such a microscopic approach should contain a series of consecutive, strictly controlled steps. In particular, based on the individual equations of motion for the structural units of the system, the Liouville equation must be obtained for the distribution function of all the particles of the system. From the Liouville equation, the transition must be made to the equations of motion for many-particle distribution functions, which will have the form 
of an infinite chain of kinetic equations, which, in essence, is a generalization of the BBGKY chain to the case of systems with active fluctuations. The next step in the procedure for constructing a kinetic theory is the controlled truncation of this chain in any perturbation theory. In the present article, as the small parameters of such a perturbation theory, we choose the weak interaction between the structural units of the system and the low intensity of the external stochastic effect on it. In this case, the density of the system is not necessarily small, and the closed kinetic equation for the single-particle distribution function has the form of the Fokker-Planck equation. Recall that, in accordance with [23], it is also possible in perturbation theory to break an infinite chain of equations - an analogue of the BBGKY chain and in the case of low density of the medium with arbitrary interaction between particles, if only this interaction does not lead to the formation of bound states. In this case, we would inevitably come to some analogue of the Boltzmann kinetic equation. However, for the active media under study, the possible (in principle) implementation of this case leads to significant mathematical difficulties and requires separate efforts (see this in regards Ref. [31]). Note also that in the transition from the Liouville equation to an infinite chain of equations, an additional intermediate step is required. It is connected with the necessity of averaging the Liouville equation, obtained from the initial equations of motion for structural units, over an external stochastic field. In this case, the experience of work [30] described above becomes useful, the technique of which is easily modified in relation to the systems studied in this article.

Building a consistent microscopic approach requires, first of all, to go beyond the Langevin equations as the initial equations of motion for the structural units of the system (see in this regard [30]). Instead of the Langevin equations, it is necessary to formulate the generalized Hamilton equations, that is, to base the first principles of classical theoretical mechanics. It is within the framework of such formulations that it is possible to most correctly include the interaction between structural units, both potential and dissipative. In this case, there are no problems with the inclusion of velocity-dependent noise correlated both in time and in coordinate. Note that the proposed theory does not deny the possibility of incorporating the Langevin equations as the initial equations of motion for structural units of the active medium. However, it will not be possible to talk about a rigorous microscopic theory that comes from first principles. In fact, the Langevin equations themselves were originally obtained as phenomenological equations of motion (Newton's equations), in which the force of the medium on a selected (test) particle is divided into a regular friction force and some random force. Thus, the Langevin equations are formulated for obviously noninteracting particles. For this reason, the inclusion of interaction between particles in these equations is again associated with the involvement of phenomenological approaches. Note also that in the "traditional" Langevin equations, the noise acting on Brownian particles depends only on time, but not on the coordinates. The presence of spatially correlated noise in the Langevin equations in the existing methods for deriving the Fokker - Planck kinetic equation would lead to serious mathematical difficulties. Thus, 
in accordance with the requirements listed above, the task of describing kinetic processes in the systems under study is posed in the most general form possible.

The solution of this problem in the present work is also carried out in the most general and consistent form, adhering to the first principles of statistical physics. First of all, this refers to the derivation of general kinetic equations for a system of active particles. Of course, a number of successive simplifications were used in the paper, connected with the transition from more complex systems, for which more complex equations were derived, to simpler systems. In particular, we completely refused in the present work the generalization of the proposed approach in the case of non-Gaussian noise. First of all, it was done for the sake of reducing the volume of the article. In addition, such a simplification is possible for the reason that, in the light of [30], the ways of modifying the approach proposed in this article to the case of non-Gaussian noise seem quite understandable and do not contain fundamental difficulties. The paper also carried out a fairly detailed analysis of some more simple specific examples, designed to demonstrate the effectiveness of the proposed method and the kinetic equations obtained in terms of the implementation of the announced goals. Such efficiency is most easily illustrated by analyzing cases in which the results of our theory coincide or are close to the results of other authors obtained earlier in other approaches. Section 6 of this paper is fully devoted to the solution of the latter problem, in which the results of the analysis of some particular solutions of our equations are given.

Thus, as the result of the consistent implementation of the above tasks, we suggest a generalized formulation of the reduced description method, suitable for describing the kinetics of many-particle dissipative systems with active fluctuations. It is shown that in the framework of the microscopic approach developed it is possible to construct the kinetic theory of active particles both in the cases of two- and three-dimensional systems, with the availability of non-linear friction (dissipative interaction), as well as local impact of an external active random field. Under the local impact we assume that this field may act differently at different points in space. In other words, the effect of this field on a particle may depend not only on the velocity (or momentum) of that specific particle, but on the point in the coordinate space where the particle is located. The general kinetic equations for such systems are obtained. We also consider special cases in which the obtained kinetic equations give solutions known for the active particles from the earlier works [3, 18, 20, 21].

\section{Basics}

Consider a system consisting of $N$ identical active particles of mass $m$, each of which is characterized by spatial coordinates $\mathbf{x}_{\alpha}, 1 \leq \alpha \leq N$, measured from the center of mass, and momentum $\mathbf{p}_{\alpha}, 1 \leq \alpha \leq N$. The interaction between the particles is assumed to consist of two parts - a "reversible" part described by the Hamiltonian $H$, and "irreversible" one, described by the function $R^{\omega}$, the meaning of which will be explained below. 
The Hamiltonian of the system can be written as:

$$
H=H_{0}+V=\sum_{1 \leq \alpha \leq N} \frac{\mathbf{p}_{\alpha}^{2}}{2 m}+\sum_{1 \leq \alpha<\beta \leq N} V_{\alpha, \beta},
$$

where $V_{\alpha, \beta}$ is the pair interaction potential,

$$
\begin{aligned}
& V_{\alpha, \beta} \equiv V\left(\mathbf{x}_{\alpha \beta}\right), \\
& \mathbf{x}_{\alpha \beta} \equiv \mathbf{x}_{\alpha}-\mathbf{x}_{\beta} .
\end{aligned}
$$

We also assume that the particles of the system are exposed to specific forces that depend on the particle velocity (or momentum) and are characterized by a function $R$. We assume that the function $R$ can be represented as:

$$
R=R^{r}+R^{\omega}
$$

where $R^{r}$ is a regular part of this function.

$$
\begin{aligned}
& R^{r} \equiv \sum_{1 \leq \alpha<\beta \leq N} R_{\alpha, \beta}, \\
& R_{\alpha, \beta} \equiv R\left(\mathbf{x}_{\alpha \beta}, \mathbf{p}_{\alpha \beta}\right), \\
& \mathbf{p}_{\alpha \beta} \equiv \mathbf{p}_{\alpha}-\mathbf{p}_{\beta},
\end{aligned}
$$

and $R^{\omega}$ is a stochastic part of the function $R$, which can be written as

$$
R^{\omega} \equiv \sum_{1 \leq \alpha \leq N} R^{\omega}\left(x_{\alpha}, t\right), x_{\alpha} \equiv\left\{\mathbf{x}_{\alpha}, \mathbf{p}_{\alpha}\right\}
$$

The stochastic nature of the function $R^{\omega}$ is formally highlighted by the presence of index $\omega$.

Note that in the case of non-active identical particles with the dissipative interaction function, $R^{r}$ is treated as a dissipative function, see [32] and [30, 31]. It is usually assumed that the dissipation in the system is related to friction of macroscopic particles, so that in this case the dissipation function $R_{\alpha, \beta}$, following [32], can be chosen as:

$$
R_{\alpha, \beta} \equiv \frac{1}{2} \tilde{\gamma}\left(\mathbf{x}_{\alpha \beta}\right) \mathbf{p}_{\alpha \beta}^{2}, \quad \tilde{\gamma}\left(\mathbf{x}_{\alpha \beta}\right)>0, \quad \mathbf{p}_{\alpha \beta} \equiv \mathbf{p}_{\alpha}-\mathbf{p}_{\beta} .
$$

This implies that $\tilde{\gamma}\left(\mathbf{x}_{\alpha \beta}\right)=0$ if $\left|\mathbf{x}_{\alpha \beta}\right|>r_{0}$, where $r_{0}$ is a characteristic range of dissipative forces. In view of the property (5) the friction coefficient is always positive.

However, in case of the active particles the positivity does not always hold [3]. The friction coefficient in the Langevin-type equations for active particles can depend on the velocity and change its sign. Therefore, one can not use the criteria (5) to determine the properties of dissipation function $R_{\alpha, \beta}$ in the case of active particles.

Following the usual classical theoretical mechanics procedures, and taking into account the Eqs. (1) - (4), the generalized Hamilton equations for the system under study can be written as

$$
\dot{\mathbf{p}}_{\alpha}=-\frac{\partial H}{\partial \mathbf{x}_{\alpha}}-\frac{\partial R}{\partial \mathbf{p}_{\alpha}}, \quad \dot{\mathbf{x}}_{\alpha}=\frac{\partial H}{\partial \mathbf{p}_{\alpha}} .
$$




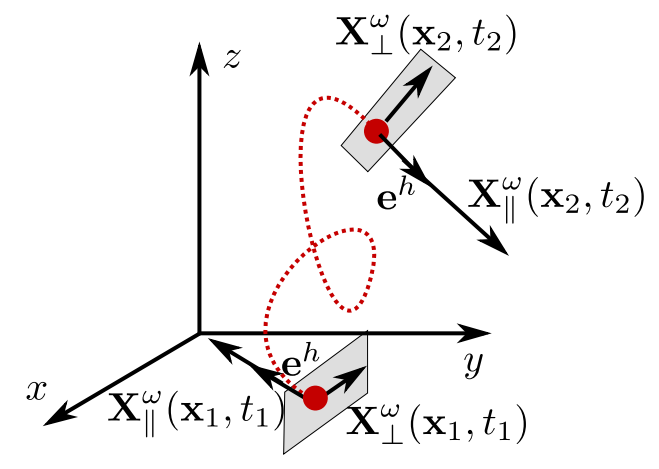

Figure 1. Schematic visualization of particle motion in the presence of stochastic effects with components $X_{\| i}^{\omega}\left(\mathbf{x}_{\alpha}, \mathbf{p}_{\alpha}, t\right), X_{\perp i}^{\omega}\left(\mathbf{x}_{\alpha}, \mathbf{p}_{\alpha}, t\right)$ according to Eq. (9)

Thus, the force $\mathbf{F}_{\alpha, \beta}$ acting on a particle $\alpha$ from the particle $\beta$ consists of two terms:

$$
\mathbf{F}_{\alpha, \beta}=\mathbf{F}_{\alpha, \beta}^{p}+\mathbf{F}_{\alpha, \beta}^{r},
$$

namely, the force $\mathbf{F}_{\alpha, \beta}^{p}$, connected with the presence of a potential pair interaction between the particles, and the force $\mathbf{F}_{\alpha, \beta}^{r}$, connected with the presence of a dissipative interaction between the particles (in the sense outlined above)

$$
\mathbf{F}_{\alpha, \beta}^{p} \equiv-\frac{\partial V_{\alpha, \beta}}{\partial \mathbf{x}_{\alpha}}, \quad \mathbf{F}_{\alpha, \beta}^{r} \equiv-\frac{\partial R_{\alpha, \beta}}{\partial \mathbf{p}_{\alpha}} .
$$

In addition, the $\alpha$-th particle is influenced by external random force $\mathbf{Y}_{\alpha}^{\omega}$ (see Eq. (1), which depends on the momentum of the particle, wherein:

$$
\begin{aligned}
- & \frac{\partial R\left(x_{\alpha}, t\right)}{\partial p_{i \alpha}} \equiv Y_{i}^{\omega}\left(x_{\alpha}, t\right)=X_{\| i}^{\omega}\left(\mathbf{x}_{\alpha}, \mathbf{p}_{\alpha}, t\right)+X_{\perp i}^{\omega}\left(\mathbf{x}_{\alpha}, \mathbf{p}_{\alpha}, t\right) \\
X_{\| i}^{\omega}\left(\mathbf{x}_{\alpha}, \mathbf{p}_{\alpha}, t\right) & \equiv X^{\omega}\left(\mathbf{x}_{\alpha}, t\right) e_{\alpha i}^{h}, \quad X_{\perp i}^{\omega}\left(\mathbf{x}_{\alpha}, \mathbf{p}_{\alpha}, t\right) \equiv X_{j}^{\omega}\left(\mathbf{x}_{\alpha}, t\right)\left(\delta_{i j}-e_{\alpha i}^{h} e_{\alpha j}^{h}\right), \quad e_{\alpha i}^{h} \equiv \frac{p_{\alpha i}}{\left|\mathbf{p}_{\alpha}\right|} .
\end{aligned}
$$

The latter expression requires some comments. We emphasize, first of all, that the stochastic force $Y_{i}^{\omega}\left(x_{\alpha}, t\right)$ in Eq. (9) is written in a form that is not related to the choice of a particular coordinate system. This notation simply reflects the fact that the stochastic force acts differently along and across the direction of a particle velocity. Fig. 1 shows a schematic visualization of the assumption written in the form (9). The expression (9) looks like a natural extension of the stochastic force $Y_{i}^{\omega}(\mathbf{x}, t)$ typical for the Langevin equation in case of an ordinary Brownian particle:

$$
\dot{\mathbf{x}}=\mathbf{v}, \quad \dot{v}_{i}=-\gamma v_{i}+Y_{i}^{\omega}(\mathbf{x}, t) .
$$

In fact, the value $Y_{i}^{\omega}(\mathbf{x}, t)$ in the last equation can always be identically rewritten as:

$$
Y_{i}^{\omega}=\left(\mathbf{e}^{h} \mathbf{Y}^{\omega}\right) e_{i}^{h}+Y_{j}^{\omega}\left(\delta_{i j}-e_{i}^{h} e_{j}^{h}\right),
$$

where $e_{i}^{h}$ is arbitrary unit vector, for example, $e_{i}^{h}=p_{i} /|\mathbf{p}|$. Replacing here the scalar product $\mathbf{e}^{h} \mathbf{Y}^{\omega}$ with $X^{\omega}\left(\mathbf{x}_{\alpha}, t\right), Y_{j}^{\omega}$ by $X_{j}^{\omega}\left(\mathbf{x}_{\alpha}, t\right)$ and assuming $e_{\alpha i}^{h}=p_{\alpha i} /\left|\mathbf{p}_{\alpha}\right|$, we arrive at Eq. (9). It should be remembered, however, that in Eq. (9) the values $X^{\omega}\left(\mathbf{x}_{\alpha}, t\right)$, $X_{j}^{\omega}\left(\mathbf{x}_{\alpha}, t\right)$ do not relate to each other, in general. If necessary, in the three-dimensional case the vector $X_{j}^{\omega}\left(\mathbf{x}_{\alpha}, t\right)$ can be considered as two-component in a plane perpendicular 
to the vector $e_{\alpha j}^{h}$. The presence of components $X_{j}^{\omega}\left(\mathbf{x}_{\alpha}, t\right)$ along $e_{\alpha j}^{h}$ will not affect the description of processes and phenomena in such systems in any case because of the factor $\left(\delta_{i j}-e_{\alpha i}^{h} e_{\alpha j}^{h}\right)$ in the right-hand side of Eq. (9).

It follows from the above that the stochastic effects on the system under consideration in the form of Eq. (9) can be regarded as a generalization of stochastic forces used in the theory of two-dimensional systems of active particles, i.e. active fluctuations, see, e.g., Refs. [3], [18]. First, Eq. (9) allows for the possibility of local influence of stochastic forces on the system. Second, Eq. (9) can be applied both to the two- and three-dimensional systems as well. To see this it is sufficient to consider the expression (9) two-dimensional and non-local, put it

$\mathbf{e}_{\alpha}^{h} \equiv \mathbf{e}_{h}, X^{\omega}(t) \equiv \sqrt{D_{v}} \xi_{v}(t), \quad \mathbf{X}^{\omega}\left(\mathbf{x}_{\alpha}, t\right) \equiv \mathbf{e}_{\phi} \sqrt{D_{\phi}} \xi_{\phi}(t), \quad \mathbf{e}_{h} \mathbf{e}_{\phi}=0$,

where $\mathbf{e}_{h}$ is a unit vector along the direction of motion of a particle, $\mathbf{e}_{\phi}$ is a unit vector along the azimuthal angle $\phi$ and $D_{\phi}, D_{v}$ are angular and velocity noise intensities, respectively [3]. Note that in two-dimensional systems, as is known, the isolated directions can appear in the movement of active particles. The special importance of the direction of the particles motion, set by its orientation vector (head-tail axis) is due to the existence of the propulsion mechanism. Thus, due to the head-tail asymmetry in the steady state of the many active particles system it is possible to fix the reference system in a natural way by a special choice of the vectors $\mathbf{e}_{h}, \mathbf{e}_{\phi}$. The existence of this asymmetry is reflected in the many-particle system characteristics, such as a oneparticle distribution function. As it is shown below, the existence of the effects of headtail asymmetry is also possible in three dimensions, even in the case of a linear friction (see Section 5 of this paper). We emphasize that the source of stochastic effects can be generalized to three dimensions in another, as compared with Eq. (9), form. Similar to the two-dimensional case, one can use, e.g., the spherical coordinates. However, in this paper it is easier to employ the Cartesian coordinates.

Let us also note the following. The time derivative of the total energy of the system in accordance with the Eqs. (1), (6) is given by

$$
\frac{d H}{d t}=-\sum_{1 \leq \alpha \leq N} \frac{\mathbf{p}_{\alpha}}{m} \frac{\partial R}{\partial \mathbf{p}_{\alpha}} .
$$

If we assume that the system has a dissipation due to friction of macroscopic particles, and a regular part of the dissipation function $R^{r}$ is given by Eq. (5), then Eq. (11) together with Eq. (9) give

$\frac{d H}{d t}=-\frac{2}{m} \sum_{1 \leq \alpha<\beta \leq N} R_{\alpha, \beta}+\sum_{1 \leq \alpha \leq N} \frac{p_{i \alpha}}{m}\left[X^{\omega}\left(\mathbf{x}_{\alpha}, t\right) e_{\alpha i}^{h}+X_{j}^{\omega}\left(\mathbf{x}_{\alpha}, t\right)\left(\delta_{i j}-e_{\alpha i}^{h} e_{\alpha j}^{h}\right)\right]$,

or

$$
\frac{d H}{d t}=-\frac{2}{m} \sum_{1 \leq \alpha<\beta \leq N} R_{\alpha, \beta}+\sum_{1 \leq \alpha \leq N} \frac{\left|\mathbf{p}_{\alpha}\right|}{m} X^{\omega}\left(\mathbf{x}_{\alpha}, t\right) .
$$

Taking into account that $\tilde{\gamma}\left(\mathbf{x}_{\alpha \beta}\right)>0$, see Eq. (5), in such a system competition between the dissipation due to friction and the pumping of energy from the stochastic field is possible. 
Further task is to obtain the Liouville equation. To this end, for the convenience of further calculations we represent the equations (6) in the following form

$$
\dot{x}_{\alpha}(t)=h_{\alpha}^{\omega}\left(x_{1}(t), \ldots, x_{N}(t)\right), \quad 1 \leq \alpha \leq N,
$$

where we introduce the notation

$$
x_{a}(t) \equiv\left(\mathbf{x}_{a}(t), \mathbf{p}_{a}(t)\right) .
$$

Thus, Eq. (13) together with Eqs. (14), (5) read

$$
\dot{\mathbf{x}}_{\alpha}(t)=\mathbf{h}_{\mathbf{x} \alpha}^{\omega}(x(t)), \quad \dot{\mathbf{p}}_{\alpha}(t)=\mathbf{h}_{\mathbf{p} \alpha}^{\omega}(x(t)),
$$

where

$$
\mathbf{h}_{\mathbf{x} \alpha}^{\omega}(x(t))=\frac{\partial H}{\partial \mathbf{p}_{\alpha}}, \quad \mathbf{h}_{\mathbf{p} \alpha}^{\omega}(x(t))=-\frac{\partial H}{\partial \mathbf{x}_{\alpha}}-\frac{\partial R}{\partial \mathbf{p}_{\alpha}} .
$$

The coordinates and momenta of $\alpha$-th particle at time $t$ (see Eq. (14)), are determined by the coordinates and momenta $x_{0} \equiv\left(x_{1}(0), \ldots, x_{N}(0)\right)$ of all the particles at the initial time $t=0$ :

$$
x_{\alpha}^{\omega}(t)=X_{\alpha}^{\omega}\left(t, x_{0}\right) \equiv\left(\mathbf{X}_{\alpha}^{\omega}\left(t, x_{0}\right), \mathbf{P}_{\alpha}^{\omega}\left(t, x_{0}\right)\right),
$$

where the functions $\mathbf{X}_{\alpha}^{\omega}\left(t, x_{0}\right), \mathbf{P}_{\alpha}^{\omega}\left(t, x_{0}\right)$ satisfy the generalized Hamilton equations (5) (or equations $(13)-(16))$. Introduce the probability density $D\left(x_{1}(0), \ldots, x_{N}(0) ; 0\right)$ of the initial conditions $x_{0} \equiv\left(x_{1}(0), \ldots, x_{N}(0)\right)$,

$$
\int d x_{1}(0) \ldots d x_{N}(0) D\left(x_{1}(0), \ldots, x_{N}(0) ; 0\right) \equiv \int d x_{0} D\left(x_{0} ; 0\right)=1 .
$$

Then, at time $t$ the probability density $D^{\omega}\left(x_{1}, \ldots, x_{N} ; t\right) \equiv D^{\omega}(x ; t), x \equiv\left(x_{1}, \ldots, x_{N}\right)$, $(N$-particle distribution function) is defined by the expression

$$
D^{\omega}\left(x_{1}, \ldots, x_{N} ; t\right)=\int d x_{0} D\left(x_{0} ; 0\right) \prod_{1 \leq \alpha \leq N} \delta\left(x_{\alpha}-X_{\alpha}^{\omega}\left(t, x_{0}\right)\right) .
$$

In [33] a detailed procedure for the obtaining of the Liouville equation for many-particle systems in an external stochastic field neglecting the interaction between the particles is described. In [30] (see also [33]), a similar procedure is used to obtain a generalized Liouville equation for dissipative many-particle systems in the absence of an external stochastic fields. The $N$-particle distribution function obeys the continuity equation

$$
\frac{\partial D^{\omega}}{\partial t}+\sum_{1 \leq \alpha \leq N} \frac{\partial}{\partial x_{\alpha}}\left(D^{\omega} h_{\alpha}^{\omega}\right)=0
$$

where the function $h_{\alpha}^{\omega}(x(t))$ is given by the expressions (16), (17). This is the Liouville equation generalized to the case of active particles with pair interactions under the influence of external stochastic fields depending on the velocities of the particles. With Eqs. (13) - (16) it can be written as:

$$
\frac{\partial D^{\omega}}{\partial t}+\sum_{1 \leq \alpha \leq N} \frac{\partial}{\partial \mathbf{x}_{\alpha}}\left(D^{\omega} \frac{\partial H}{\partial \mathbf{p}_{\alpha}}\right)+\sum_{1 \leq \alpha \leq N} \frac{\partial}{\partial \mathbf{p}_{\alpha}}\left(D^{\omega}\left(-\frac{\partial H}{\partial \mathbf{x}_{\alpha}}-\frac{\partial R}{\partial \mathbf{p}_{\alpha}}\right)\right)=0
$$


In what follows we will use the Liouville equation (21), transformed with Eqs. (13) (16), (8), and (9) to the form

$\frac{\partial D^{\omega}}{\partial t}+\sum_{1 \leq \alpha \leq N} \frac{\mathbf{p}_{\alpha}}{m} \frac{\partial D^{\omega}}{\partial \mathbf{x}_{\alpha}}+\sum_{1 \leq \alpha<\beta \leq N} \frac{\partial}{\partial \mathbf{p}_{\alpha}} D^{\omega} \mathbf{F}_{\alpha, \beta}+\sum_{1 \leq \alpha \leq N} \frac{\partial}{\partial \mathbf{p}_{\alpha}} D^{\omega} \mathbf{Y}_{\alpha}^{\omega}=0$

where $\mathbf{F}_{\alpha, \beta}, \mathbf{Y}_{\alpha}^{\omega}$ are determined by Eqs. (7) - (9). Equation (22) is an example of the evolution equation with multiplicative noise. Now, the goal is to average this equation over realizations of the external random force $\mathbf{Y}_{\alpha}^{\omega}$.

\section{Averaging generalized Liouville equation with Gaussian random force}

We introduce the $N$-particle distribution function $D\left(x_{1}, \ldots, x_{N} ; t\right)$, which is the distribution function $D^{\omega}\left(x_{1}, \ldots, x_{N} ; t\right)$ (see Eq. (19), averaged over the random external field $\mathbf{Y}^{\omega}(\mathbf{x}, t)$ with probability density $W\left[\mathbf{Y}^{\omega}\right]$ :

$D\left(x_{1}, \ldots, x_{N} ; t\right) \equiv\left\langle D^{\omega}\left(x_{1}, \ldots, x_{N} ; t\right)\right\rangle_{\omega}, \quad\langle\ldots\rangle_{\omega} \equiv \int D \mathbf{Y}^{\omega}(\mathbf{x}, t) W\left[\mathbf{Y}^{\omega}\right] \ldots$

Using the averaging operation (23) for the equation (22), we obtain:

$\frac{\partial D}{\partial t}+\sum_{1 \leq \alpha \leq N} \frac{\mathbf{p}_{\alpha}}{m} \frac{\partial D}{\partial \mathbf{x}_{\alpha}}+\sum_{1 \leq \alpha<\beta \leq N} \frac{\partial}{\partial \mathbf{p}_{\alpha}} D \mathbf{F}_{\alpha, \beta}+\sum_{1 \leq \alpha \leq N} \frac{\partial}{\partial \mathbf{p}_{\alpha}}\left\langle D^{\omega} \mathbf{Y}_{\alpha}^{\omega}\right\rangle_{\omega}=0$.

To have a closed evolution equation for the distribution function introduced, it is necessary to express the value of $\left\langle D^{\omega} \mathbf{Y}_{\alpha}^{\omega}\right\rangle_{\omega}$ through $D\left(x_{1}, \ldots, x_{N} ; t\right)$. We use the so-called Furutsu-Novikov formula [34, 35], which was proved for the case of Gaussian distributions of the external random field. For non-Gaussian random fields the FurutsuNovikov formula is generalized in [29] (see also [30]). In this article, we will not recount the latter proof, referring to the works cited above. We use the result of such a proof of [29] in the case of a Gaussian distribution of multiplicative noise. Thus, we get

$$
\begin{aligned}
\left\langle Y_{i}^{\omega}\left(x_{\alpha}, t\right) D^{\omega}\right. & {\left.\left[\mathbf{Y}^{\omega}\right]\right\rangle_{\omega}=Y_{i}\left(x_{\alpha}, t\right)\left\langle D^{\omega}\left[\mathbf{Y}^{\omega}\right]\right\rangle_{\omega} } \\
& +\int d x^{\prime} \int_{-\infty}^{\infty} d t^{\prime} y_{i j}\left(x_{\alpha}, x^{\prime}, t-t^{\prime}\right)\left\langle\frac{\delta D^{\omega}\left[\mathbf{Y}^{\omega}\right]}{\delta Y_{j}\left(x^{\prime}, t^{\prime}\right)}\right\rangle_{\omega}
\end{aligned}
$$

where $Y_{i}\left(x_{\alpha}, t\right) \equiv\left\langle Y_{i}^{\omega}\left(x_{\alpha}, t\right)\right\rangle_{\omega}, x_{\alpha} \equiv\left\{\mathbf{x}_{\alpha}, \mathbf{p}_{\alpha}\right\}$ and $y_{i j}\left(x_{\alpha}, x^{\prime}, t-t^{\prime}\right)$ is a pair correlation function of the external Gaussian noise $\left(x^{\prime} \equiv\left\{\mathbf{x}^{\prime}, \mathbf{p}^{\prime}\right\}\right)$ :

$y_{i j}\left(x_{\alpha}, x^{\prime}, t-t^{\prime}\right)=\left\langle Y_{i}^{\omega}\left(x_{\alpha}, t\right) Y_{j}^{\omega}\left(x^{\prime}, t^{\prime}\right)\right\rangle_{\omega}-\left\langle Y_{i}^{\omega}\left(x_{\alpha}, t\right)\right\rangle_{\omega}\left\langle Y_{j}^{\omega}\left(x^{\prime}, t^{\prime}\right)\right\rangle_{\omega}$.

In what follows we use $Y_{i}(x) \equiv 0$. Now, let us consider

$$
I_{i} \equiv \int d x^{\prime} \int_{-\infty}^{\infty} d t^{\prime} y_{i j}\left(x_{\alpha}, x^{\prime}, t-t^{\prime}\right)\left\langle\frac{\delta D^{\omega}\left[\mathbf{Y}^{\omega}\right]}{\delta Y_{j}^{\omega}\left(x^{\prime}, t^{\prime}\right)}\right\rangle_{\omega},
$$

in more detail. We assume that the pair correlation function $y_{i j}\left(x_{\alpha}, x^{\prime}, t-t^{\prime}\right)$ is different from zero in the interval $\left|t-t^{\prime}\right| \leq \tau_{0}$. We also assume that when $t \sim t^{\prime}$, pair correlation function $y_{i j}\left(x_{\alpha}, x^{\prime}, t-t^{\prime}\right)$ has a sharp maximum. Then the functional derivative $\frac{\delta D^{\omega}\left[\mathbf{Y}^{\omega}\right]}{\delta Y_{j}^{\omega}\left(x^{\prime}, t^{\prime}\right)}$ is to be evaluated only at $t \approx t^{\prime}$. Moreover, as shown in [29, 34, 35, 36], an exact expression for this derivative can be obtained only when $t \approx t^{\prime}$. 
In fact, the variational derivative $\frac{\delta D^{\omega}\left[\mathbf{Y}^{\omega}\right]}{\delta Y_{j}^{\omega}\left(x^{\prime}, t^{\prime}\right)}$ at $t \approx t^{\prime}$ undergoes a jump:

$$
\frac{\delta D^{\omega}\left[\mathbf{Y}^{\omega}\right]}{\delta \delta Y_{j}^{\omega}\left(x^{\prime}, t^{\prime}\right)} \neq 0, \quad t^{\prime} \leq t, \quad \frac{\delta D^{\omega}\left[\mathbf{Y}^{\omega}\right]}{\delta Y_{j}^{\omega}\left(x^{\prime}, t^{\prime}\right)}=0, \quad t^{\prime}>t
$$

The latter circumstance is due to the fact that according to the equation (22), the value of $D^{\omega}(t)$ can not depend on the field $Y_{j}^{\omega}\left(\mathbf{x}^{\prime}, t^{\prime}\right)$ taken at a later time than $t$. According to Eq. (27) the integration over $t^{\prime}$ in the formula (27) is held in the range of $-\infty$ to $t$, instead of $-\infty$ to $+\infty$.

Differentiating Eq. (22) by $Y j^{\omega}\left(\mathbf{x}^{\prime}, t^{\prime}\right)$ and noting that according to Eq. (27) the derivative $\frac{\partial}{\partial t} \frac{\delta D^{\omega}\left[\mathbf{Y}^{\omega}\right]}{\delta Y_{j}^{\omega}\left(x^{\prime}, t^{\prime}\right)}$ must have a $\delta$-like shape in time (while the value $\frac{\delta D^{\omega}\left[\mathbf{Y}^{\omega}\right]}{\delta Y_{j}^{\omega}\left(x^{\prime}, t^{\prime}\right)}$ does not), the following expression for the functional derivative is obtained (see [29]):

$$
\begin{aligned}
& \frac{\delta D^{\omega}\left[\mathbf{Y}^{\omega}\right]}{\delta Y_{j}^{\omega}\left(x^{\prime}, t^{\prime}\right)} \approx-\vartheta\left(t-t^{\prime}\right) \sum_{1 \leq \beta \leq N} \delta\left(x^{\prime}-x_{\beta}\right) \frac{\partial D^{\omega}\left[\mathbf{Y}^{\omega}\right]}{\partial p_{\beta j}}, \\
& \delta\left(x^{\prime}-x_{\beta}\right) \equiv \delta\left(\mathbf{x}^{\prime}-\mathbf{x}_{\beta}\right) \delta\left(\mathbf{p}^{\prime}-\mathbf{p}_{\beta}\right)
\end{aligned}
$$

where $\vartheta\left(t-t^{\prime}\right)$ is the Heaviside function. This formula allows us to represent $I_{i}$, Eq. (27), in the following form (see Eqs. (23), (24):

$$
I_{i}=\int_{-\infty}^{t} d t^{\prime} \sum_{1 \leq \beta \leq N} y_{i j}\left(x, x_{\beta} ; t-t^{\prime}\right) \frac{\partial D}{\partial p_{\beta j}} .
$$

Thus, the averaged Liouville equation, generalized to the case of systems of many particles with active interaction reads

$$
\begin{aligned}
\frac{\partial D}{\partial t}+\sum_{1 \leq \alpha \leq N} \frac{\mathbf{p}_{\alpha}}{m} \frac{\partial D}{\partial \mathbf{x}_{\alpha}}+\sum_{1 \leq \alpha<\beta \leq N} \frac{\partial D \mathbf{F}_{\alpha, \beta}}{\partial \mathbf{p}_{\alpha}}+\sum_{1 \leq \alpha \leq N} \frac{\partial D \mathbf{Y}\left(x_{\alpha}, t\right)}{\partial \mathbf{p}_{\alpha}} \\
-\int_{-\infty}^{t} d t^{\prime} \sum_{1 \leq \alpha, \beta \leq N} \frac{\partial}{\partial p_{\alpha i}} y_{i j}\left(x_{\alpha}, x_{\beta} ; t-t^{\prime}\right) \frac{\partial D}{\partial p_{\beta j}}=0 .
\end{aligned}
$$

Taking into account that the pair correlation function $y_{i j}\left(x_{\alpha}, x_{\beta} ; t-t^{\prime}\right)$ has a sharp maximum at $t \approx t^{\prime}$, and also assuming that this function is an even function of difference $t-t^{\prime}$

$$
y_{i j}\left(x_{\alpha}, x_{\beta} ; t-t^{\prime}\right)=y_{i j}\left(x_{\alpha}, x_{\beta} ; t^{\prime}-t\right),
$$

then Eq. (31) gets even a simpler form,

$$
\begin{aligned}
\frac{\partial D}{\partial t}+\sum_{1 \leq \alpha \leq N} \frac{p_{\alpha}}{m} \frac{\partial D}{\partial \mathbf{x}_{\alpha}}+\sum_{1 \leq \alpha<\beta \leq N} \frac{\partial D \mathbf{F}_{\alpha, \beta}}{\partial \mathbf{p}_{\alpha}} \\
-\frac{1}{2} \sum_{1 \leq \alpha, \beta \leq N} \frac{\partial}{\partial p_{\alpha i}} y_{i j}\left(x_{\alpha}, x_{\beta}\right) \frac{\partial D}{\partial p_{\beta j}}=0
\end{aligned}
$$

where we introduce the notation:

$$
y_{i j}\left(x_{\alpha}, x_{\beta}\right) \equiv \int_{-\infty}^{\infty} d \tau y_{i j}\left(x_{\alpha}, x_{\beta} ; \tau\right) .
$$


Equation (33) can be put in another form suitable for further calculations:

$$
\begin{aligned}
\frac{\partial D}{\partial t}+\sum_{1 \leq \alpha \leq N} \frac{\mathbf{p}_{\alpha}}{m} & \frac{\partial D}{\partial \mathbf{x}_{\alpha}}+\sum_{1 \leq \alpha<\beta \leq N} \frac{\partial D \mathbf{F}_{\alpha, \beta}}{\partial \mathbf{p}_{\alpha}} \\
& -\frac{1}{2} \sum_{1 \leq \alpha \leq N} \frac{\partial}{\partial p_{\alpha i}} y_{i j}\left(x_{\alpha}, x_{\alpha}\right) \frac{\partial D}{\partial p_{\alpha j}}-\sum_{1 \leq \alpha<\beta \leq N} \frac{\partial}{\partial p_{\alpha i}} y_{i j}\left(x_{\alpha}, x_{\beta}\right) \frac{\partial D}{\partial p_{\beta j}}=0 .
\end{aligned}
$$

Note that, in fact, the developed technique allows to obtain a generalized Liouville equation also in case of non-Gaussian random field whenever these distributions have moments of any order, see [29]. In the present paper, however, we restrict ourselves to a Gaussian external random field.

For further calculations, we specify the explicit form of the pair correlation function $y_{i j}\left(x_{\alpha}, x_{\beta}\right)$. Using Eqs. (9) and Eq. (26) we arrive at the following expression for $y_{i j}\left(x_{\alpha}, x_{\beta}\right)$ :

$$
\begin{aligned}
& y_{i j}\left(x_{\alpha}, x_{\beta}\right)=e_{i \alpha}^{h} e_{j \beta}^{h} g\left(\mathbf{x}_{\alpha}, \mathbf{x}_{\beta}\right)+\left(\delta_{i \ell}-e_{i \alpha}^{h} e_{\ell \alpha}^{h}\right)\left(\delta_{j \ell}-e_{j \beta}^{h} e_{\ell \beta}^{h}\right) h\left(\mathbf{x}_{\alpha}, \mathbf{x}_{\beta}\right), \\
& e_{\alpha i}^{h}=\frac{p_{i \alpha}}{\left|\mathbf{p}_{\alpha}\right|},
\end{aligned}
$$

where we introduced the notations:

$$
\begin{aligned}
g\left(\mathbf{x}_{\alpha}, \mathbf{x}_{\beta}\right) \equiv & \int_{-\infty}^{\infty} d t\left\langle X^{\omega}\left(\mathbf{x}_{\alpha}, t\right) X^{\omega}\left(\mathbf{x}_{\beta}, t^{\prime}\right)\right\rangle_{\omega}, \\
& \delta_{\ell k} h\left(\mathbf{x}_{\alpha}, \mathbf{x}_{\beta}\right) \equiv \int_{-\infty}^{\infty} d t\left\langle X_{\ell}^{\omega}\left(\mathbf{x}_{\alpha}, t\right) X_{k}^{\omega}\left(\mathbf{x}_{\beta}, t^{\prime}\right)\right\rangle_{\omega} .
\end{aligned}
$$

When obtaining expressions (35) we assumed that the stochastic force $Y_{i}^{\omega}(x, t)$ has the following properties:

$$
\left\langle X^{\omega}\left(\mathbf{x}_{\alpha}, t\right) X_{i}^{\omega}\left(\mathbf{x}_{\beta}, t^{\prime}\right)\right\rangle=0, \quad\left\langle X^{\omega}\left(\mathbf{x}_{\alpha}, t\right)\right\rangle=0, \quad\left\langle X_{i}^{\omega}\left(\mathbf{x}_{\beta}, t^{\prime}\right)\right\rangle=0 .
$$

The last two formulas in Eq. (37) are the result of the requirement $Y_{i}(x, t) \equiv$ $\left\langle Y_{i}^{\omega}(x, t)\right\rangle_{\omega}=0$, see Eq. (9).

\section{Analogue of the BBGKY chain for systems of identical active particles interacting with external random fields}

Along with the probability density $D\left(x_{1}, \ldots, x_{N}, t\right)$ we can introduce the probability of finding one or more particles in the given elements of phase space, regardless of the positions of the remaining particles (see also [22, 23]). These probabilities can be obtained by integrating the function $D$ over all variables except those that relate to the particles under consideration:

$f_{S}\left(x_{1}, \ldots, x_{S} ; t\right)=\mathcal{V}^{S} \int d x_{S+1} \ldots \int d x_{N} D\left(x_{1}, \ldots, x_{N} ; t\right), \quad x_{\alpha} \equiv\left(\mathbf{x}_{\alpha}, \mathbf{p}_{\alpha}\right)$,

where $D\left(x_{1}, \ldots, x_{N} ; t\right)$ satisfies Eq. (34) and $\mathcal{V}$ is the system volume. Following the procedure described in $[37,38]$, after some transformations we arrive at the following 
equation for the $S$-particle distribution function $f_{S}\left(x_{1}, \ldots, x_{S} ; t\right)$ :

$$
\begin{aligned}
\frac{\partial f_{S}}{\partial t}+\sum_{1 \leq \alpha \leq S} \frac{\mathbf{p}_{\alpha}}{m} & \frac{\partial f_{S}}{\partial x_{\alpha}}-\frac{1}{2} \sum_{1 \leq \alpha \leq S} \frac{\partial}{\partial p_{\alpha i}} y_{i j}\left(x_{\alpha}, x_{\alpha}\right) \frac{\partial f_{S}}{\partial p_{\alpha j}} \\
& +\sum_{1 \leq \alpha<\beta \leq S} \frac{\partial f_{S} \mathbf{F}_{\alpha, \beta}}{\partial \mathbf{p}_{\alpha}}-\sum_{1 \leq \alpha<\beta \leq S} \frac{\partial}{\partial p_{\alpha i}} y_{i j}\left(x_{\alpha}, x_{\beta}\right) \frac{\partial f_{S}}{\partial p_{\beta j}}= \\
& -\frac{1}{\mathfrak{v}} \sum_{1 \leq \alpha \leq S} \frac{\partial}{\partial \mathbf{p}_{\alpha}} \int d x_{S+1} f_{S+1} \mathbf{F}_{\alpha, S+1} \\
& +\frac{1}{\mathfrak{v}} \sum_{1 \leq \alpha \leq S} \frac{\partial}{\partial p_{\alpha i}} \int d x_{S+1} y_{i j}\left(x_{\alpha}, x_{S+1}\right) \frac{\partial f_{S+1}}{\partial p_{S+1 j}}, \quad \mathfrak{v} \equiv \frac{\mathcal{V}}{N},
\end{aligned}
$$

where the quantities $\mathbf{F}_{\alpha, \beta}$ and $y_{i j}\left(x_{\alpha}, x_{\beta}\right)$ are still given by Eqs. (8), (26), (35) and (36). As it is easy to see the equation for the $S$-particle distribution function includes a $S+1$-particle distribution function. Thus, in fact, we obtain an infinite chain of kinetic equations (39). These chains are a generalization of the well-known chain of Bogolyubov-Born-Green-Kirkwood-Yvon equations in case of identical active interacting particles under the influence of external stochastic fields. It is necessary to make the following remark. According to the definition (38), the distribution functions of a higher order contain all the information contained in the functions of lower order [22]. This leads to the fact that with the increase in the order $S$, the distribution functions $f_{S}\left(x_{1}, \ldots, x_{S} ; t\right)$ are becoming increasingly complex. Since in full description according to Eq. (39) it is necessary to consider the distribution functions up to $S=N$, we conclude that the resulting chain of equations (39) themselves are equivalent to Liouville equation (34). In other words, the most complete description of the studied systems is equally complex both within the framework of the full the distribution function $D\left(x_{1}, \ldots, x_{N} ; t\right)$, and the one of the many-particle distribution functions $f_{S}\left(x_{1}, \ldots, x_{S} ; t\right)$.

A significant simplification in description of the state of the system occurs in two cases: when the interaction between the particles is small, or when the number density of particles is small, and the interaction is arbitrary, but is such that does not lead to the formation of bound states [38]. This simplification in the description is the consequence of the difference in the evolutionary behaviour of a many- and a singleparticle distribution functions. In fact, at an early stage of evolution, when the time $t$ is small compared to the characteristic time of chaotization $\tau_{0}$, the multi-particle distribution functions $f_{S}\left(x_{1}, \ldots, x_{S} ; t\right)$ change rapidly over time, in contrast to the singleparticle distribution function $f_{1}(x, t)$. Single-particle distribution function experiences significant changes in time at times much longer than the relaxation time of the system $\tau_{r}$, and $\tau_{r} \gg \tau_{0}$. Time $\tau_{0}$, in order of magnitude is determined by the duration of one collision. It can also be estimated as a value of the order of $r_{c} / \bar{v}$, where $\bar{v}$ is the average velocity of the particles and $r_{c}$ is the correlation radius, that is, the distance from which the multi-particle distribution functions break up into a single-particle product. Usually, the order of magnitude of the correlation radius coincides with the radius of action of the forces, that is why $\tau_{0}$ can be considered of the order of the duration of one collision 
(see also $[22,23]$ in this regard). While time $\tau_{r}$ in order of magnitude should be the same as the time of the establishment of statistical equilibrium state in the system (for more details see Ref. [23]). Such difference in the evolutionary behaviour of the single-particle and many-particle distribution functions formed the basis of the ideas of Bogolyubov about a hierarchy of the system relaxation times [22].

According to the idea of a hierarchy of relaxation times, the evolution of manyparticle system can be divided into several stages. Each subsequent stage of evolution differs from the previous by a simplification in the description of the evolution of system of many particles. The simplest scenario for the evolution of systems of many particles is as follows. When $\tau_{0} \ll t \ll \tau_{r}$ there takes place a kinetic stage of evolution of the system, when the system behaviour can be described by a single-particle distribution function. This description of the system evolution is much easier than that using the multi-particle distribution functions. Further simplification of the description of many-particle systems occurs when $t \gg \tau_{r}$ (the hydrodynamic stage of evolution of the system), when the behaviour of the system can be described by the hydrodynamic description parameters, for example, the particle number density, the average velocity and the temperature of the medium. Such a gradual simplification of the system description the approaches of the reduced description method are based on [22, 23].

In this paper, the method of reduced description of non-equilibrium processes will be used for the derivation of the kinetic equations describing the evolution of systems of interacting active particles in an external random field. The initial equations will be the chain equations (39). The mathematical formulation of the idea of a hierarchy of relaxation times of the system is a time-functional dependence of manyparticle distribution functions $f_{S}\left(x_{1}, \ldots, x_{S} ; t\right)$ only through a dependence on time of the parameters of the reduced description at the appropriate stage of evolution. In particular, at the kinetic stage of the evolution the many-particle distribution functions depend on time only through the one-particle distribution function $f_{1}\left(x^{\prime}, t\right)$ :

$$
f_{S}\left(x_{1}, \ldots, x_{S} ; t\right)=f_{S}\left(x_{1}, \ldots, x_{S} ; f_{1}\left(x^{\prime}, t\right)\right) .
$$

In addition to the functional hypothesis (40), the reduced description method is also based on the principle of spatial correlation weakening. In the language of multiparticle distribution functions, this principle can be summarized as follows [23]. Let $S$ of the particles can be divided into two sub-groups of particles containing $S^{\prime}$ and $S^{\prime \prime}$ particles, respectively, $S=S^{\prime}+S^{\prime \prime}$. If the distance $R$ between these subgroups of particles increases infinitely, $R \rightarrow \infty$, then due to the weakening of correlations between particles the $S$-particle distribution function decomposes into the product of the distribution functions related to the each particles sub-group:

$$
f_{S}\left(x_{1}, \ldots, x_{S} ; t\right) \underset{R \rightarrow \infty}{\rightarrow} f_{S^{\prime}}\left(x_{1}^{\prime}, \ldots, x_{S}^{\prime} ; t\right) f_{S^{\prime \prime}}\left(x_{1}^{\prime \prime}, \ldots, x_{S}^{\prime \prime} ; t\right) .
$$

In Eq. (41) the sign of "prime" is used to indicate the coordinates and momenta of the particles of the subgroup $S^{\prime}$, and "two primes" to indicate the coordinates and momenta of the particles of the second subgroup. It should be noted, however, 
that the principle of spatial correlation weakening Eq. (41) refers to the many-particle distribution functions, for which the thermodynamic limit is made $N \rightarrow \infty, \mathcal{V} \rightarrow \infty$, and $(N / \mathcal{V})=$ const $[38]$.

According to Eq. (40), the time derivative of $\partial f_{S} / \partial t$ in Eq. (39) when $S \neq 1$ must be understood as follows:

$$
\frac{\partial}{\partial t} f_{S}\left(x_{1}, \ldots, x_{S} ; f_{1}(x, t)\right)=\int d x^{\prime} \frac{\delta f_{S}\left(x_{1}, \ldots, x_{S} ; f_{1}(x, t)\right)}{\delta f_{1}\left(x^{\prime}, t\right)} \frac{\partial f_{1}\left(x^{\prime}, t\right)}{\partial t},
$$

where $\delta f_{S}\left(f_{1}(x, t)\right) / \delta f_{1}\left(x^{\prime}, t\right)$ is the functional derivative. The single-particle distribution function itself $f_{1}\left(x^{\prime}, t\right)$ according to Eq. (39) must satisfy the equation:

$$
\frac{\partial f_{1}}{\partial t}+\frac{\mathbf{p}_{1}}{m} \frac{\partial f_{1}}{\partial \mathbf{x}_{1}}-\frac{1}{2} \frac{\partial}{\partial p_{1 i}} y_{i j}\left(x_{1}, x_{1}\right) \frac{\partial f_{1}}{\partial p_{1 j}}=\frac{1}{\mathfrak{v}} L\left(x_{1} ; f_{1}\right),
$$

where as before $\mathfrak{v}=\mathcal{V} / N$ and $L\left(x_{1} ; f_{1}\right)$ is the generalized collision integral defined by the formula

$$
\begin{aligned}
L\left(x_{1} ; f_{1}\right) \equiv- & \frac{\partial}{\partial \mathbf{p}_{1}} \int d x_{2} f_{2}\left(x_{1}, x_{2} ; f_{1}\right) \mathbf{F}_{1,2} \\
& +\frac{\partial}{\partial p_{\alpha i}} \int d x_{2} y_{i j}\left(x_{1}, x_{2}\right) \frac{\partial}{\partial p_{2 j}} f_{2}\left(x_{1}, x_{2} ; f_{1}\right) .
\end{aligned}
$$

As is easily seen to close the equation (43), one must obtain the collision integral (44) as a functional of particle distribution function for what it is necessary to cut off an infinite chain of equations (39). Clearly, this can only be done within a certain approximation. In particular, in the system of non-active particles such cutoff may be implemented in the two cases mentioned above, when the interaction between the particles is small or when the particle density is low, and the interaction is arbitrary but such that does not lead to the formation of bound states [22]. Similar situations can be implemented in the case of a system of identical active particles with interaction, which is discussed in this paper. We will demonstrate this in the case of the weak interaction of all kinds between the active particles and the external noise of low intensity. In other words, we assume the forces $\mathbf{F}_{\alpha, \beta}$ and the correlation functions of an external random field are small.

First, however, we make some remarks. Functional relation (40) does not necessarily imply an expansion of $f_{S}\left(x_{1}, \ldots, x_{S} ; f_{1}\left(x^{\prime}, t\right)\right)$ in functional perturbation series by the one-particle distribution function. This expansion must be realized only in one of the above-mentioned cases of chain breaking, namely when the particles density is low. We remind that thus arises the famous question of the possible divergences in higher orders of perturbation theory by a small particle number density and about a renormalization of this theory (see, e.g., [39, 40, 41]). In the case of perturbation theory by the weak interaction between the particles, these issues do not appear, as is easily seen from the subsequent calculations (see also [23], [29]). 


\section{Kinetic equations for systems of weakly interacting active particles in external random field of low intensity}

Here we follow the methodology suggested in [23]. Using Eqs. (42), (43), a chain of equations (39) can be written as:

$$
-\int d x \frac{\delta f_{S}\left(f_{1}\right)}{\delta f_{1}(x, t)} \frac{\mathbf{p}}{m} \frac{\partial f_{1}(x, t)}{\partial \mathbf{x}}+\sum_{1 \leq \alpha \leq S} \frac{\mathbf{p}_{\alpha}}{m} \frac{\partial f_{S}\left(f_{1}\right)}{\partial \mathbf{x}_{\alpha}}=\frac{1}{\mathfrak{v}} K_{S}\left(f_{1}\right),
$$

where

$$
\begin{aligned}
K_{S}\left(f_{1}\right) \equiv-\mathfrak{v} & \sum_{1 \leq \alpha<\beta \leq S} \frac{\partial f_{S} \mathbf{F}_{\alpha, \beta}}{\partial \mathbf{p}_{\alpha}}+\frac{1}{2} \sum_{1 \leq \alpha \leq S} \frac{\partial}{\partial p_{\alpha i}} y_{i j}\left(x_{\alpha}, x_{\alpha}\right) \frac{\partial f_{S}}{\partial p_{\alpha j}} \\
& +\sum_{1 \leq \alpha<\beta \leq S} \frac{\partial}{\partial p_{\alpha i}} y_{i j}\left(x_{\alpha}, x_{\beta}\right) \frac{\partial f_{S}}{\partial p_{\beta j}}-\sum_{1 \leq \alpha \leq S} \frac{\partial}{\partial \mathbf{p}_{\alpha}} \int d x_{S+1} f_{S+1} \mathbf{F}_{\alpha, S+1} \\
& +\sum_{1 \leq \alpha \leq S} \frac{\partial}{\partial p_{\alpha i}} \int d x_{S+1} y_{i j}\left(x_{\alpha}, x_{S+1}\right) \frac{\partial f_{S+1}}{\partial p_{S+1 j}} \\
& -\int d x_{1} \frac{\delta f_{S}\left(f_{1}\right)}{\delta f_{1}\left(x_{1}, t\right)}\left\{L\left(x_{1} ; f_{1}\right)+\frac{1}{2} \frac{\partial}{\partial p_{1 i}} y_{i j}\left(x_{1}, x_{1}\right) \frac{\partial f_{1}\left(x_{1}, t\right)}{\partial p_{1 j}}\right\} .
\end{aligned}
$$

The chain of equations (45), (46) must be supplemented by the "initial conditions". To this end, following $[22,23]$, we introduce an auxiliary parameter $\tau$, having the dimension of time, but does not necessarily representing the physical time. We next consider the many-particle distribution function $f_{S}\left(\mathbf{x}_{1}-\frac{\mathbf{p}_{1}}{m} \tau, \mathbf{p}_{1}, \ldots, \mathbf{x}_{S}-\frac{\mathbf{p}_{S}}{m} \tau, \mathbf{p}_{S} ; f_{1}\right)$. According to Eq. (41) this function must satisfy the asymptotic relation:

$f_{S}\left(\mathbf{x}_{1}-\frac{\mathbf{p}_{1}}{m} \tau, \mathbf{p}_{1}, \ldots, \mathbf{x}_{S}-\frac{\mathbf{p}_{S}}{m} \tau, \mathbf{p}_{S} ; f_{1}\right) \underset{\tau \rightarrow \infty}{\rightarrow} \prod_{1 \leq \alpha \leq S} f_{1}\left(\mathbf{x}_{\alpha}-\frac{\mathbf{p}_{\alpha}}{m} \tau, \mathbf{p}_{\alpha}\right)$.

If we define further the shift operator $\hat{\Lambda}_{S}^{0}$ in the coordinate space with the formula

$$
i \hat{\Lambda}_{S}^{0} \equiv \sum_{1 \leq \alpha \leq S} \frac{\mathbf{p}_{\alpha}}{m} \frac{\partial}{\partial \mathbf{x}_{\alpha}}
$$

the condition (47) may be rewritten as:

$$
e^{i \tau \hat{\Lambda}_{S}^{0}} f_{S}(\tau) \underset{\tau \rightarrow \infty}{\rightarrow} \prod_{1 \leq \alpha \leq S} f_{1}\left(x_{\alpha}\right)
$$

where $\exp \left(i \tau \hat{\Lambda}_{S}^{0}\right)$ is a so-called "free evolution operator" and

$$
f_{S}(\tau) \equiv f_{S}\left(x_{1}, \ldots, x_{S} ; e^{-i \tau \hat{\Lambda}_{1}^{0}} f_{1}\left(x^{\prime}\right)\right)=f_{S}\left(x_{1}, \ldots, x_{S} ; f_{1}\left(\mathbf{x}^{\prime}-\frac{\mathbf{p}^{\prime}}{m} \tau, \mathbf{p}^{\prime}\right)\right) .
$$

Now, Eq. (45) can be written in the following way:

$$
\frac{\partial}{\partial \tau} e^{i \tau \hat{\Lambda}_{S}^{0}} f_{S}(\tau)=\frac{1}{\mathfrak{v}} e^{i \tau \hat{\Lambda}_{S}^{0}} K_{S}(\tau)
$$

where

$$
K_{S}(\tau) \equiv K_{S}\left(x_{1}, \ldots, x_{S} ; e^{-i \tau \hat{\Lambda}_{1}^{0}} f_{1}\left(x^{\prime}\right)\right)=K_{S}\left(x_{1}, \ldots, x_{S} ; f_{1}\left(\mathbf{x}^{\prime}-\frac{\mathbf{p}^{\prime}}{m} \tau, \mathbf{p}^{\prime}\right)\right) .
$$


Integrating equation (51) over $\tau$ within the limits from $-\infty$ to 0 and using the asymptotic conditions (49), we get

$$
f_{S}\left(x_{1}, \ldots, x_{S} ; f_{1}\left(x^{\prime}\right)\right)=\prod_{1 \leq \alpha \leq S} f_{1}\left(x_{\alpha}\right)+\frac{1}{\mathfrak{v}} \int_{-\infty}^{0} d \tau e^{i \tau \hat{\Lambda}_{S}^{0}} K_{S}(\tau) .
$$

The ratio (53) allow to develop a perturbation theory in the weak interaction and the intensity of stochastic effects. Under such assumptions, the value $K_{S}(\tau)$ (see Eq. (47) can be considered small, and therefore, in the main approximation we have

$$
f_{S}\left(x_{1}, \ldots, x_{S} ; f_{1}\left(x^{\prime}\right)\right)=\prod_{1 \leq \alpha \leq S} f_{1}\left(x_{\alpha}\right)
$$

which implies

$$
f_{2}\left(x_{1}, x_{2}\right)=f_{1}\left(x_{1}\right) f_{1}\left(x_{2}\right) .
$$

Substituting further Eq. (54) into Eq. (44) and using Eqs. (7), (8), we obtain the following closed kinetic equation:

$$
\begin{aligned}
\frac{\partial f_{1}\left(x_{1}, t\right)}{\partial t}+ & \frac{\mathbf{p}_{1}}{m} \frac{\partial f_{1}\left(x_{1}, t\right)}{\partial \mathbf{x}_{1}}-\frac{1}{2} \frac{\partial}{\partial p_{1 i}} y_{i j}\left(x_{1}, x_{1}\right) \frac{\partial f_{1}\left(x_{1}, t\right)}{\partial p_{1 j}} \\
& =\frac{1}{\mathfrak{v}} \frac{\partial}{\partial \mathbf{p}_{1}} f_{1}\left(x_{1}\right) \int d x_{2} f_{1}\left(x_{2}\right)\left(\frac{\partial V_{1,2}}{\partial \mathbf{x}_{1}}+\frac{\partial R_{1,2}}{\partial \mathbf{p}_{1}}\right) \\
& +\frac{1}{\mathfrak{v}} \frac{\partial}{\partial p_{1 i}} f_{1}\left(x_{1}\right) \int d x_{2} y_{i j}\left(x_{1}, x_{2}\right) \frac{\partial f_{1}\left(x_{2}\right)}{\partial p_{2 j}},
\end{aligned}
$$

where the values $V_{1,2}, R_{1,2}$ are given by Eqs. (2) - (4) and the correlation function $y_{i j}\left(x_{1}, x_{2}\right)$ is still given by Eq. (35). Equation (55) and can be rewritten in a slightly different form:

$$
\begin{aligned}
\frac{\partial f_{1}\left(x_{1}, t\right)}{\partial t}+ & \frac{\mathbf{p}_{1}}{m} \frac{\partial f_{1}\left(x_{1}, t\right)}{\partial \mathbf{x}_{1}}-\frac{\partial U\left(\mathbf{x}_{1}, t\right)}{\partial \mathbf{x}_{1}} \frac{\partial f_{1}\left(x_{1}, t\right)}{\partial \mathbf{p}_{1}} \\
& =\frac{1}{2} \frac{\partial}{\partial p_{1 i}} y_{i j}\left(x_{1}, x_{1}\right) \frac{\partial f_{1}\left(x_{1}, t\right)}{\partial p_{1 j}} \\
& +\frac{1}{\mathfrak{v}} \frac{\partial}{\partial p_{1 i}} f_{1}\left(x_{1}, t\right) \int d x_{2} f_{1}\left(x_{2}, t\right)\left[\frac{\partial R_{1,2}}{\partial p_{1 i}}-\frac{\partial y_{i j}\left(x_{1}, x_{2}\right)}{\partial p_{2 j}}\right]
\end{aligned}
$$

or

$$
\begin{aligned}
\frac{\partial f_{1}\left(x_{1}, t\right)}{\partial t}+ & \frac{\mathbf{p}_{1}}{m} \frac{\partial f_{1}\left(x_{1}, t\right)}{\partial \mathbf{x}_{1}}-\frac{\partial U\left(\mathbf{x}_{1}, t\right)}{\partial \mathbf{x}_{1}} \frac{\partial f_{1}\left(x_{1}, t\right)}{\partial \mathbf{p}_{1}} \\
& =\frac{1}{2} \frac{\partial}{\partial p_{1 i}} y_{i j}\left(x_{1}, x_{1}\right) \frac{\partial f_{1}\left(x_{1}, t\right)}{\partial p_{1 j}} \\
& +\frac{1}{\mathfrak{v}} \frac{\partial}{\partial p_{1 i}} f_{1}\left(x_{1}, t\right) \int d x_{2}\left[R_{1,2} \delta_{i, j}+y_{i j}\left(x_{1}, x_{2}\right)\right] \frac{\partial f_{1}\left(x_{2}, t\right)}{\partial p_{2 j}}
\end{aligned}
$$

if we consider an average field $U\left(\mathbf{x}_{1}, t\right)$, defined by the formula (see Eq. (2):

$U\left(\mathbf{x}_{1}, t\right)=\frac{1}{\mathfrak{v}} \int d \mathbf{x}_{2} V\left(\mathbf{x}_{1}-\mathbf{x}_{2}\right) \int d \mathbf{p}_{2} f_{1}\left(x_{2}, t\right), \quad f_{1}\left(x_{2}\right) \equiv f_{1}\left(\mathbf{x}_{2}, \mathbf{p}_{2}\right)$.

Equations (55) - (57) are the kinetic equations for the active particles with pair interactions (potential and "dissipative" ones) between the particles under the influence 
of active space-dependent fluctuations. We emphasize that all the equations (55) (57) are obtained without using the explicit form of the potential interaction $V_{1,2} \equiv$ $V\left(\mathbf{x}_{1}-\mathbf{x}_{2}\right)$, dissipation function $R_{12}$, and the correlation function $y_{i j}\left(x_{1}, x_{2}\right)$.

Note that the presence of a random force (9), typical for active fluctuations and having a local effect on the particles leads, as it is seen from Eqs. (56), (57) to an additional interaction between particles, determined by the pair correlation function $y_{i j}\left(x_{1}, x_{2}\right)$.

We also note the following circumstance. The basis for obtaining equations (55) (58) is formula (54), which reflects the main approximation of the perturbation theory developed in the paper for small interactions between particles, including dissipative interaction, as well as for low external noise intensity. Naturally, formula (54), indicating that there are no correlations between particles, is already understood at the intuitive level. After all, it is clear that, in this form, the two-particle distribution function allows one to truncate the BBGKY chain and obtain a closed kinetic equation for the one-particle distribution function. However, writing this formula at once, assuming that due to the small interactions and the weakness of the stochastic effects, the correlations between the particles can be neglected, thereby completely ignoring the material of Section 5 to equations (55) - (58), would be wrong for several reasons. First, it is not obvious. One need to know what may be neglected. Especially, that the purpose of the present study, as mentioned above, is the announced development of a common microscopic approach based on first principles of statistical physics to the construction of the kinetic theory of dissipative media with active fluctuations like it was done in $[22,23,24,25,26,27]$ with a preference for strict approaches and formulations of the monograph [23]. This means that the scheme for constructing such a microscopic approach should contain a number of consecutive, strictly controlled steps, including with respect to truncating the BBGKY chain. Secondly, the scheme given in Section 5 allows, if necessary, to obtain amendments to the right-hand side of formula (54), firstorder terms for interactions and weak stochastic processes. Thus, expressions would be obtained that determine the correlations between the particles of the medium in the first approximation of perturbation theory. It is important to note that in equations (55) - (57) there would appear terms quadratic in the interactions (see in this regard [31]) and cross terms, that is, multiplicative in interactions and characteristics of stochastic influence. However, we considered that within the framework of this paper it is better to refrain from considering such effects. First of all, in order not to clutter an article with material that is not necessary from the point of view of the main tasks announced in it. Nevertheless, it should be recognized that the discussed terms may be responsible for very interesting effects, for example, related to the above-mentioned effect of the appearance of additional interaction between structural units of the environment. Currently, the authors are studying the issues raised here, which, as it turned out, along with analytical methods require the involvement of completely non-trivial numerical methods. For this reason, the analysis of the issue requires additional time.

In addition, we make a few more comments at the end of this section. We have 
already emphasized above the reasons why we refused in this article to generalize the developed approach to the case of non-Gaussian noise. Here, in connection with this generalization, we draw attention to the fact that when comparing the materials of this work and [30], the mathematical similarity of the derivation procedures for chains of equations (analogs of BBGKY chains) for many-particle distribution functions and kinetic equations for single-particle distribution functions is noteworthy. The similarity of the noted procedures indicates the possibility of "organic" generalization of the approach of the present work in the case of non-Gaussian noise. Such a task will not be of principal difficulties, but it will greatly clutter up the calculations and significantly worsen the visibility of the results. In this connection, the external mathematical similarity of some parts of this article and of the Ref. [30] in the particular case of the Gaussian stochastic effect on the system is also noteworthy. In particular, we are talking about the similarity of the mathematical structure of equations (34) and (57) of this work and, accordingly, equations (40), (63) from [30]. Such a similarity should be considered formal if we take into account the fact that this article and Ref. [30] deal with completely different systems. In fact, equations (34) and (57) of this article describe dissipative systems with nonlinear (in general) friction and active fluctuations. This is reflected in the dependence of the pair correlation functions included in (34), (57), not only from the coordinates, but also from the momenta (or velocities), which, as noted above, is characteristic of such systems. Equations (40), (63) from [30] describe the evolution of dissipative systems with nonlinear (in general) friction and a source of stochastic noise, depending only on coordinates. A similar circumstance characterizes the system studied in the [30] as a dissipative system with passive fluctuations.

\section{Particular cases for spatially homogeneous systems}

Thus, we have developed microscopic approach to the description of the kinetics of the active media that allowed to come to common kinetic equations (55) - (57) in the approximation of the average or mean field, see (7), (8), (58). The general nature of the obtained kinetic equations must be confirmed by demonstrating the facts that in some cases these equations lead to results obtained by other authors in other approaches or for other models of active media.

Here we demonstrate that the kinetic equations (55) - (57) involve some known special cases for systems of active particles. For this, we selected examples that would give results known from earlier works by other authors, using primarily phenomenological approaches to solving such problems. In this case, it is necessary to introduce a number of successive simplifications into the obtained general equations (55) - (57). In particular, it is necessary to proceed to the consideration of spatially homogeneous cases. In addition, we recall that in the process of exposition we allowed a further simplification of the nature of noise. Namely, from a more general form of Gaussian noise (26) passed to time-delta-correlated noise, see (33), (35) - (37). And thus, from equation (31) of a more complex form to the simpler Liouville equation (34). 


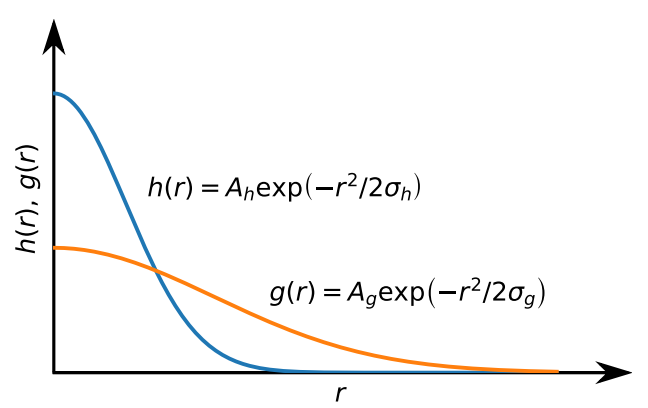

Figure 2. Schematic visualization of a simple example of different behavior of functions $g(r)$ and $h(r)$ determining the decrease of pair correlation functions with distance $r \equiv\left|\mathbf{x}_{\mathbf{1}}-\mathbf{x}_{\mathbf{2}}\right|$ according to Eq. (60)

For this reason, the path to equation (34) may seem somewhat long. After all, it would be possible to come to equation (34) more directly, immediately assuming the deltacorrelation of noise, but then we would, on the one hand, move away from controllability of the considered approximations, on the other hand, would not have a more general equation (31). So let's start by considering a spatially homogeneous state. Then, a single-particle distribution function $f_{1}(\mathbf{x}, \mathbf{p}, t)$ does not depend on the coordinates,

$$
f_{1}(\mathbf{x}, \mathbf{p}, t) \equiv f_{1}(\mathbf{p}, t) .
$$

We should note that the spatially correlated noise impacting on the system (see Eq. (9)) does not necessarily prevent the existence of the spatially homogeneous states Eq. (59). The latter are possible only in the case of a zero mean of the external random force acting on the system, and the pair correlation functions $y_{i j}\left(x_{1}, x_{2}\right)$ (see Eqs. (35) (37)) should depend only on the difference of the coordinates of the two particles,

$y_{i j}\left(x_{1}, x_{2}\right)=g\left(\mathbf{x}_{1}-\mathbf{x}_{2}\right) e_{1 i}^{h} e_{2 j}^{h}+h\left(\mathbf{x}_{1}-\mathbf{x}_{2}\right)\left(\delta_{i \ell}-e_{1 i}^{h} e_{1 l}^{h}\right)\left(\delta_{j \ell}-e_{2 j}^{h} e_{2 l}^{h}\right)$.

We remind that the assumption of a zero average of the random field was made in the present paper beginning from Eq. (32). We also emphasize that both a specific kind of functions $g\left(\mathbf{x}_{1}-\mathbf{x}_{2}\right)$ and $h\left(\mathbf{x}_{1}-\mathbf{x}_{2}\right)$, and their dependence on the coordinates difference, may be different due to uncorrelated noise components along and across the particle velocity, see Eqs. (35) - (37). As an example, this distinction is illustrated in Figure 2 for the simple case when the pair correlation functions $g\left(\mathbf{x}_{1}-\mathbf{x}_{2}\right)$ and $h\left(\mathbf{x}_{1}-\mathbf{x}_{2}\right)$ decrease with the distance $r \equiv\left|\mathbf{x}_{\mathbf{1}}-\mathbf{x}_{\mathbf{2}}\right|$ according to the Gaussian law, but with different values of the variances and amplitudes. However, it is easy to see that the calculations that led to the results of the present and subsequent sections of this work do not require the specification of the explicit form of the functions mentioned. We recall that according to Eq. (4) all restrictions on the general properties of functions $R_{1,2}$ are contained in the expression:

$$
R_{1,2} \equiv R\left(\mathbf{x}_{1}-\mathbf{x}_{2}, \mathbf{p}_{1}-\mathbf{p}_{2}\right),
$$

which follows from the Galilean invariance of the system in the absence of external influences. Moreover, since the function $R_{1,2}$ is a scalar quantity, its dependence on the 
differences $\mathbf{x}_{1}-\mathbf{x}_{2}, \mathbf{p}_{1}-\mathbf{p}_{2}$ should be characterized by the expression:

$$
R(\mathbf{x}, \mathbf{p}) \equiv R\left(\mathbf{x}^{2}, \mathbf{p}^{2}, \mathbf{x p}\right) .
$$

According to Eqs. (59) - (62) equation (55) transforms into:

$$
\begin{aligned}
& \frac{\partial f_{1}\left(\mathbf{p}_{1}, t\right)}{\partial t}-\frac{1}{2} \frac{\partial}{\partial p_{1 i}}\left[g(0) e_{1 i}^{h} e_{1 j}^{h}+h(0)\left(\delta_{i j}-e_{1 i}^{h} e_{1 j}^{h}\right)\right] \frac{\partial f_{1}\left(\mathbf{p}_{1}, t\right)}{\partial p_{1 j}} \\
& =\frac{\partial}{\partial p_{1 i}} f_{1}\left(\mathbf{p}_{1}, t\right) \frac{\partial}{\partial p_{1 i}} \int d \mathbf{p}_{2} f_{1}\left(\mathbf{p}_{2}, t\right) \bar{R}\left(\left(\mathbf{p}_{1}-\mathbf{p}_{2}\right)^{2}\right) \\
& +\frac{\partial}{\partial p_{1 i}} f_{1}\left(\mathbf{p}_{1}, t\right) \int d \mathbf{p}_{2}\left[\bar{g} e_{1 i}^{h} e_{2 j}^{h}+\bar{h}\left(\delta_{i \ell}-e_{1 i}^{h} e_{1 l}^{h}\right)\left(\delta_{j \ell}-e_{2 j}^{h} e_{2 l}^{h}\right)\right] \\
& \times \frac{\partial f_{1}\left(\mathbf{p}_{2}, t\right)}{\partial p_{2 j}}
\end{aligned}
$$

where we introduce

$$
\begin{aligned}
\bar{R}\left(\left(\mathbf{p}_{1}-\mathbf{p}_{2}\right)^{2}\right) & \equiv \frac{1}{\mathfrak{v}} \int d \mathbf{x} R\left(\mathbf{x}^{2},\left(\mathbf{p}_{1}-\mathbf{p}_{2}\right)^{2}, \mathbf{x}\left(\mathbf{p}_{1}-\mathbf{p}_{2}\right)\right) \\
\bar{g} & \equiv \frac{1}{\mathfrak{v}} \int d \mathbf{x} g(\mathbf{x}) \\
\bar{h} & \equiv \frac{1}{\mathfrak{v}} \int d \mathbf{x} h(\mathbf{x}) .
\end{aligned}
$$

Brownian particles with active fluctuations. Space-independent noise Here we study solutions of the kinetic equations (63), which are isotropic in the momentum space.

$$
f_{1}(\mathbf{p}, t) \equiv f_{1}(p, t)
$$

Taking Eqs. (64), (65) it is possible to reduce Eq. (63) to the form:

$$
\begin{aligned}
\frac{\partial f_{1}\left(p_{1}, t\right)}{\partial t}= & \frac{\partial}{\partial p_{1 i}} e_{1 i}^{h}\left\{f_{1}\left(p_{1}, t\right) \gamma\left(p_{1}, t\right) p_{1}+\frac{1}{2} g(0) \frac{\partial f_{1}\left(p_{1}, t\right)}{\partial p_{1}}\right. \\
& \left.+\bar{g} f_{1}\left(p_{1}, t\right) \int d \mathbf{p}_{2} \frac{\partial f_{1}\left(p_{2}, t\right)}{\partial p_{2}}\right\}
\end{aligned}
$$

where

$$
\begin{aligned}
& \gamma(p, t) \equiv 2 \frac{\partial \bar{R}\left(p^{2}, t\right)}{\partial p^{2}} \\
& \bar{R}\left(p^{2}, t\right) \equiv \int d \mathbf{p}_{2} f_{1}\left(p_{2}, t\right) \bar{R}\left(\left(\mathbf{p}-\mathbf{p}_{2}\right)^{2}\right)
\end{aligned}
$$

The resulting equation (66) is the kinetic equation for active particles with timedependent non-linear friction (friction factor $\gamma(p, t)$ ). This equation can be regarded as a three-dimensional generalization of the kinetic equation for quasi-Brownian particles with active fluctuations, dissipative interaction and space-dependent external stochastic field. This fact may be proven if we make some simplifications of Eq. (66).

First of all, note, that the term "Brownian particles with active fluctuations" is commonly understood as a system of particles under the influence of a space-independent stochastic field given by Eqs. (9) and (10), see [3, 21]. Consequently, to prove 
the above assumption, we should pass to the linear friction case in Eq. (66) and refuse the dependence of the external noise on the coordinates. In case of a linear friction the friction coefficient $\gamma(p)$ does not depend on the momentum, $\gamma(p) \equiv \gamma$, and, according to Eqs. (5), (64), the value of $\gamma$ in this case is given by (see [30]):

$$
\gamma=\frac{1}{\mathfrak{v}^{2}} \int d \mathbf{x} \tilde{\gamma}(\mathbf{x}), \quad \int d \mathbf{p} f_{1}(p, t)=\frac{1}{\mathfrak{v}} .
$$

However, the consequences of the noise space-independence in Eq. (66) are rather hard to see immediately. For this we need to repeat the whole procedure of the kinetic equation derivation until Eqs. (55) - (57), assuming that the values $X^{\omega}(\mathbf{x}, t), X_{j}^{\omega}(\mathbf{x}, t)$ in Eq. (9) are independent of the coordinates, and the conditions (35) - (37) are fulfilled. It turns out, that the result of this procedure is equivalent to equating the value $\bar{g}$ in Eq. (66) to zero, so that we come to the following equation:

$$
\begin{aligned}
& \frac{\partial f_{1}\left(p_{1}, t\right)}{\partial t}=\frac{\partial}{\partial p_{1 i}} e_{1 i}^{h}\left\{\gamma p_{1} f_{1}\left(p_{1}, t\right)+D_{p} \frac{\partial f_{1}\left(p_{1}, t\right)}{\partial p_{1}}\right\} \\
& g(0) \equiv 2 D_{p}=2 m^{2} D_{v} .
\end{aligned}
$$

The quantity $g(0) \equiv 2 D_{p}$ is still defined by the relations Eqs. (36) and (60), keeping in mind the fact that the noise characteristics $X^{\omega}(\mathbf{x}, t)$ does not depend on the coordinate in this case. If in Eq. (69) we pass from the particles' momentum distribution function $f_{1}(p, t)$ to the distribution function in the velocity, $f_{1}(v, t), \mathbf{p}=m \mathbf{v}$, then the equation takes the form usual for the case of quasi-Brownian particles with active fluctuations, see, e.g. [3, 20, 21]. At the same time, the second formula in Eq. (69) connects intensity of the "momentum" noise $D_{p}$ introduced here with intensity of the "velocity" noise $D_{v}$, see Eqs. (9) - (10). This implies that Eq. (66) may be regarded as a kinetic equation for quasi-Brownian particles with active fluctuations, which is generalized for the case of a 3D system with dissipative interaction and a non-local external stochastic field.

The stationary solution $f_{\infty}(p)=\lim _{t \rightarrow \infty} f_{1}(p, t)$ of Eq. (69) has a Boltzmann form,

$$
f_{\infty}(p)=A \exp \left(-\frac{\gamma}{2 D_{p}} p^{2}\right)
$$

which is different in a $2 \mathrm{D}$ and $3 \mathrm{D}$ cases only by the value of the normalizing constant $A$, see Eq. (68):

$$
\begin{aligned}
A & =\frac{\gamma}{2 \pi D_{p} \mathfrak{v}} & \text { for } & 2 D \\
A & =\frac{1}{\mathfrak{v}}\left(\frac{2 \pi D_{p}}{\gamma}\right)^{-3 / 2} & \text { for } & 3 D .
\end{aligned}
$$

Taking into account normalization (68), (71) in the two- dimensional case, the formula (70) for stationary distribution function of the active particles coincides with the corresponding expression in Ref. [3].

Brownian particles with active local fluctuations We now investigate the spatially homogeneous stationary states of the system under study, in the case of a 
spatially inhomogeneous external impact. As we already noted (see text explaining formulas (59) - (60), as well as Fig. 2), spatially correlated noise in certain cases does not prevent the existence of spatially homogeneous states in the system. Let us now consider stationary solution $f_{\infty}(p)=\lim _{t \rightarrow \infty} f_{1}(p, t)$ of Eq. (66), which is more general than Eq. (69). The former in the limit $t \rightarrow \infty$ can be written as:

$$
\frac{\partial}{\partial p_{i}} e_{i}^{h}\left\{f_{\infty}(p) \gamma(p) p+D_{p} \frac{\partial f_{\infty}(p)}{\partial p}+\tilde{g} f_{\infty}(p)\right\}=0
$$

where we introduce

$$
g(0) \equiv 2 D_{p}, \quad \tilde{g} \equiv \bar{g} \int d \mathbf{p}_{2} \frac{\partial f_{\infty}\left(p_{2}\right)}{\partial p_{2}},
$$

$\bar{g}$ is given by Eq. (64) and

$$
\gamma\left(p_{1}\right)=2 \lim _{t \rightarrow \infty} \frac{\partial \bar{R}\left(p^{2}, t\right)}{\partial p^{2}}=2 \frac{\partial}{\partial p^{2}} \int d \mathbf{p}_{2} f_{\infty}\left(p_{2}\right) \bar{R}\left(\left(\mathbf{p}-\mathbf{p}_{2}\right)^{2}\right)
$$

defines the non-linear friction forces. The solution of equation (72) is defined by the relation

$$
f_{\infty}(p) \sim \exp \left\{-\frac{1}{D_{p}} \int^{p} d p^{\prime}\left(\gamma\left(p^{\prime}\right) p^{\prime}+\tilde{g}\right)\right\} .
$$

The expression similar to Eq. (75) is given in Ref. [18] for the case of two-dimensional active systems with non-linear friction coefficient. We emphasize that in this paper, as well as in the formula in the general analysis of the above expression, the friction coefficient $\gamma(p)$ is considered to be an arbitrary function of the momentum (in fact, of the velocity $v, \gamma(v)$, since the authors of this work performed calculations in the space of velocities, not momenta.)

The general analysis of this expression in [18] with an arbitrary dependence of the coefficient of friction on velocity shows that it is the non-linearity of this coefficient that is one of the necessary conditions for the occurrence of self-propulsion in the system. Indeed, as it comes from the analysis in the case of a non-linear friction, for one velocity range the dissipation forces may be negative, while within the other velocity range, on the contrary, positive. Thus, the particles with the velocities within the first interval slow down, consequently, this interval corresponds to a regular friction. The particles with the velocities within the second interval on the contrary, accelerate, that is, they experience propulsion. As a result, a symmetric distribution with two peaks at $\pm v_{0}$ is established. We here note that the stationary distribution cannot be isotropic in the velocity (or momentum) space due to the existence of a selected direction $\mathbf{e}_{0 i}^{h}=\mathbf{v}_{0 i} / v_{0}$ in the stationary state. Such distribution corresponds to the general solution of the stationary kinetic equation derived in [18] on the basis of two-dimensional Langevin equation for active particles with non-linear friction coefficient. We also note that in [18] the case of the special dependence of the friction coefficient $\gamma(v)$ on the velocity is analyzed in detail, leading to a linear dependence on the velocity of the friction force itself, but with a shift in the velocity space. It is shown that such a case of linear friction does not prevent the appearance of self-movement in the system. As far as we know, this 
result was obtained for the first time in the mentioned work. Finally, we note that the special case of linear friction considered in [18] corresponds (both for two-dimensional and three-dimensional systems) to the case $\gamma(v) \equiv \gamma$ of linear friction in formula (75) of this work, for more details see below.

Thus, as will be shown below, it follows from the solution (75) of equation (72) that the stationary distribution function with two maxima (self-propulsion particles) can be realized also in case of a linear friction, namely, when $\gamma(p) \equiv \gamma>0$, see Eq. (68). This is due to the local impact of stochastic forces with active fluctuations. It later leads to appearance of the factor $\tilde{g}$ in Eq. (75), which is defined by the pair correlation functions of the external force and the distribution function itself. That is the reason why the solution of Eq. (72) is a complex self-consistent task, see below. In fact, the general solution in case of linear friction, as it follows from Eq. (75), is given by the formula:

$$
\begin{aligned}
& f_{\infty}(p) \sim \exp \left\{-\frac{\gamma}{2 D_{p}}\left(p+\frac{\tilde{g}}{\gamma}\right)^{2}\right\}, \\
& \tilde{g} \equiv \bar{g} \int d \mathbf{p}_{2} \frac{\partial f_{\infty}\left(p_{2}\right)}{\partial p_{2}} \\
& \bar{g} \equiv \frac{1}{\mathfrak{v}} \int d \mathbf{x} g(\mathbf{x}) .
\end{aligned}
$$

The display of the head-tail asymmetry is related to the sign of $\tilde{g}$. Namely, since $\gamma>0$, the positivity of this value, $\tilde{g}>0$, must comply with a purely dissipative case. When $\tilde{g}<0$, there are values of momenta, for which the inequality $\gamma p+\tilde{g}<0$ is true. Particles moving with corresponding momentum exhibit self-propulsion. Naturally, the very possibility of the existence of a stationary state presupposes the establishment of a stationary distribution combining both described cases. One of the possible options for achieving such a stationary state in spatially homogeneous systems is the establishment of the regime of two differently directed motions of its structural units with an average momentum $p_{0}$ along some selected direction $\mathbf{e}_{0}^{h} \equiv \mathbf{p}_{\mathbf{0}} / p_{0}$. It is this version of the stationary distribution that would correspond to the solution described in [18] for the two-dimensional case. However, the appearance of the selected direction in the momentum space indicates that the stationary state of the system ceases to be isotropic in the momentum space. For this reason, the formal construction of the corresponding stationary distribution on the basis of the solution (76) of equation (72) would be incorrect. The reason is that equation (72) was obtained from equation (66) precisely under the assumption of isotropy of the momentum space.

Therefore, in describing the stationary distribution discussed above, it is necessary to start from the most general equation (63). We recall that this equation characterizes the evolution of spatially-homogeneous states of the system. In view of the foregoing, stationary distribution under steady-state regime of multidirectional movements along one direction should be described by function

$$
f_{\infty}(\mathbf{p}) \equiv f_{\infty}\left(p_{\|}\right), \quad p_{\|} \equiv \mathbf{p} \mathbf{e}_{0}^{h}=p \mathbf{e}^{h} \mathbf{e}_{0}^{h}, \quad \mathbf{e}^{h} \equiv \mathbf{p} / p, \quad \mathbf{e}_{0}^{h} \equiv \mathbf{p}_{0} / p_{0} .
$$

We emphasize that the stationary mode defined by formulas (77) and the possibility 
of which is predicted by formulas (75), (76) is one of the possible for the system, and not the only one. In this sense, it is one of the particular stationary solutions of the general equation (63) under the assumption (77). An equation for which holds $f_{\infty}\left(p_{\|}\right)$ follows from Eq. (63) within a limit $t \rightarrow \infty$ and respecting relations (77). It has the form similar to Eq. (72) if in the latter we assume $\gamma(p) \equiv \gamma>0$ and the absolute values of momentum we replace with $p_{\|}$,

$$
\left(\gamma p_{\|}+\tilde{g}\right) f_{\infty}\left(p_{\|}\right)+D_{p} \frac{\partial f_{\infty}\left(p_{\|}\right)}{\partial p_{\|}}=0, \quad D_{p_{\|}} \equiv D_{p} \equiv \frac{1}{2} g(0) .
$$

For this reason, an analysis of the solutions of this equation can be carried out by analogy with the analysis of formulas (75) and (76) given above (see also [18]), in which the momentum absolute values to be replaced by the quantity $p_{\|}$and assume $\gamma(p) \equiv \gamma>0$.

Thus, taking into account the foregoing considerations for the case of a steadystate regime of multidirectional motions along $\mathbf{e}_{0}^{h} \equiv \mathbf{p}_{0} / p_{0}$, the stationary distribution function of active particles has the form (see, in this regard, [3, 18, 21])

$$
\begin{aligned}
f_{\infty}\left(p_{\|}\right)=C\{ & \vartheta\left(\mathbf{e}^{h} \mathbf{e}_{0}^{h}\right) \exp \left[-\frac{\gamma}{2 D_{p_{\|}}}\left(p_{\|}-p_{0}\right)^{2}\right] \\
& \left.+\vartheta\left(-\mathbf{e}^{h} \mathbf{e}_{0}^{h}\right) \exp \left[-\frac{\gamma}{2 D_{p_{\|}}}\left(p_{\|}+p_{0}\right)^{2}\right]\right\},
\end{aligned}
$$

where $\vartheta(x)$ is the Heaviside step function and $C$ is the normalization constant. Momentum $p_{0}$ in Eq. (79), characterizing the location of the maxima of the distribution function symmetric with respect to the point $p=0$ is determined by $\tilde{g}$ :

$$
p_{0}=|\tilde{g}| / \gamma \text {. }
$$

The value of $\tilde{g}$ itself, according to Eqs. (73), (76), depends on the derivative of the unknown momentum distribution function. Thus, the definition (76) with the explicit form of the distribution function (79) should be considered as an equation that connects $\tilde{g}$ to the normalization constant $C$ :

$$
\begin{aligned}
\tilde{g}=-C \frac{\gamma \bar{g}}{2 D_{p_{\|}}} & \int d \mathbf{p}\left\{\vartheta\left(\mathbf{e}^{h} \mathbf{e}_{0}^{h}\right)\left(p_{\|}-p_{0}\right) \exp \left[-\frac{\gamma}{2 D_{p_{\|}}}\left(p_{\|}-p_{0}\right)^{2}\right]\right. \\
& \left.+\vartheta\left(-\mathbf{e}^{h} \mathbf{e}_{0}^{h}\right)\left(p_{\|}+p_{0}\right) \exp \left[-\frac{\gamma}{2 D_{p_{\|}}}\left(p_{\|}+p_{0}\right)^{2}\right]\right\} .
\end{aligned}
$$

In turn, the constant $C$ is determined from the normalization condition (see Eq. (68)

$$
\int d \mathbf{p} f_{\infty}\left(p_{\|}\right)=\frac{1}{\mathfrak{v}}
$$

which can be rewritten after combining with Eq. (79) as

$$
\frac{1}{\mathfrak{v}}=C \int d \mathbf{p}\left\{\vartheta\left(\mathbf{e}^{h} \mathbf{e}_{0}^{h}\right) \exp \left[-\frac{\gamma}{2 D_{p_{\|}}}\left(p_{\|}-p_{0}\right)^{2}\right]\right.
$$




$$
\left.+\vartheta\left(-\mathbf{e}^{h} \mathbf{e}_{0}^{h}\right) \exp \left[-\frac{\gamma}{2 D_{p_{\|}}}\left(p_{\|}+p_{0}\right)^{2}\right]\right\}
$$

The latter expression is also an equation relating the constant $C$ and the unknown quantity $\tilde{g}$. Thus, the equations (81) and (82) represent a system of two equations to determine two unknown quantities, $C$ and $\tilde{g}$, in terms of parameters characterizing the system, namely friction coefficient $\gamma$, the number density of particles $1 / \mathfrak{v}$ and the parameters of noise with active fluctuations, i.e. the pair correlation function $\bar{g}$ and $g(0)=2 D_{p}$, see Eqs. (73), (76). We note that, in contrast to the magnitude of the momentum $p_{0}$, the direction $\mathbf{e}_{0}^{h} \equiv \mathbf{p}_{0} / p_{0}$ of steady motion (the "self-propulsion" motion) in the framework of a spatially homogeneous model of a medium with active particles can not be determined. To determine it, it is necessary to break the spatial isotropy of the system, for example, by introducing anisotropic interaction between particles, even infinitely small. This will also be mentioned in Conclusion. We also note that because of the integration with respect to the total volume in momentum space, equations (81), (82) have different forms for two- and three-dimensional cases. Furthermore, the calculation of the integrals over $\mathbf{p}$ in equations (81), (82) must take into account the anisotropy of the integrands in momentum space. The procedure for calculating these integrals in a spherical coordinate system in momentum space is greatly simplified if the polar axis is assumed to be directed along the vector $\mathbf{e}_{0}^{h}$, see (77).

We first consider two-dimensional case. Then Eqs. (81) and (82) take the form

$$
\begin{aligned}
& \tilde{g}=-2 \pi^{3 / 2} \sqrt{\frac{2 D_{p}}{\gamma}} \bar{g} C, \\
& \frac{1}{\mathfrak{v}}=C\left\{4 \pi \frac{D_{p}}{\gamma}+2 \pi^{3 / 2} p_{0} \sqrt{\frac{2 D_{p}}{\gamma}} \operatorname{erf}\left(p_{0} \sqrt{\frac{\gamma}{2 D_{p}}}\right)\right\}, \quad p_{0} \equiv \frac{|\tilde{g}|}{\gamma},
\end{aligned}
$$

where erf $(x)$ is the error integral:

$$
\operatorname{erf}(x) \equiv \frac{2}{\sqrt{\pi}} \int_{0}^{x} d y \exp \left(-y^{2}\right) .
$$

In general, Eqs. (83) are complex transcendental equations that can be solved numerically. However, in the two extreme cases, namely those of small and large values of the argument $p_{0} \sqrt{\gamma / 2 D_{p}}=\sqrt{\tilde{g}^{2} / 2 \gamma D_{p}}$ of the error integral (84) these equations can be solved analytically. In the case $p_{0} \sqrt{\gamma / 2 D_{p}}=\sqrt{\tilde{g}^{2} / 2 \gamma D_{p}} \ll 1$, these solutions are given by

$$
\begin{aligned}
& C \approx \frac{\gamma}{4 \pi D_{p} \mathfrak{v}}, \\
& \tilde{g}=-\pi^{1 / 2} \frac{\bar{g}}{\mathfrak{v}} \sqrt{\frac{\gamma}{2 D_{p}}} \\
& p_{0} \approx \pi^{1 / 2} \frac{1}{\mathfrak{v}} \sqrt{\frac{\bar{g}^{2}}{2 \gamma D_{p}}}
\end{aligned}
$$


With Eqs.(85) the inequality above can be written as

$$
\frac{\bar{g}}{D_{p} \mathfrak{v}} \ll 1
$$

We have already mentioned that the presence of self-proppelled properties depends on the sign of $\tilde{g}$. Since $\bar{g}$ is positive (see Eq. (76)), the quantity $\tilde{g}$ in Eq. (85) is negative, $\tilde{g}<0$. Consequently, the state like a two bell-like peaks distribution function with parameters defined by Eq. (85) can be realized.

Now consider the opposite case $p_{0} \sqrt{\gamma / 2 D_{p}}=\sqrt{\tilde{g}^{2} / 2 \gamma D_{p}} \gg 1$. Then, in the main order we get from Eq. (83):

$$
\begin{aligned}
C & \approx \frac{1}{4 \pi^{3 / 2} \mathfrak{v}} \frac{\gamma}{D_{p}} \sqrt{\frac{2 \gamma D_{p}}{\tilde{g}^{2}}}, \\
\tilde{g} & =-2 \pi^{3 / 2} \sqrt{\frac{2 D_{p}}{\gamma} \bar{g} C,} \\
p_{0} & =\sqrt{\frac{\bar{g}}{\gamma \mathfrak{v}}}
\end{aligned}
$$

We see, that also in this case the quantity $\tilde{g}$ is negative, $\tilde{g}<0$ and the particle distribution function is defined by Eqs. (79), (87). We also add that the inequality $p_{0} \sqrt{\gamma / 2 D_{p}}=\sqrt{\tilde{g}^{2} / 2 \gamma D_{p}} \gg 1$, with the use of Eq. (87) can be transformed into the relation

$$
\frac{\bar{g}}{D_{p} \mathfrak{v}} \gg 1
$$

which is the opposite to Eq. (86).

Now let us return to Eqs. (81) and (82), and study their solution in case of threedimensional system of active particles with linear friction and active space-dependent (i.e., local in space) fluctuations. In this case, Eqs. (81), (82) are tranformed to a form substantially different from Eq. (83):

$$
\begin{aligned}
& C=\frac{1}{2 v}\left(\frac{\pi \gamma}{2 D_{p}}\right)^{3 / 2}, \\
& \tilde{g}+2 \frac{|\tilde{g}|}{\gamma} \frac{\pi^{3} \bar{g}}{\mathfrak{v}} \frac{\gamma}{D_{p}} \operatorname{erf}\left(\sqrt{\frac{\tilde{g}^{2}}{2 \gamma D_{p}}}\right)=-8 \frac{\pi \bar{g} D_{p}}{\gamma \mathfrak{v}}\left(\frac{\pi \gamma}{2 D_{p}}\right)^{3 / 2}, \\
& p_{0} \equiv \frac{|\tilde{g}|}{\gamma}
\end{aligned}
$$

where erf $(x)$ is still given by Eq. (84). As in the previous case of the two-dimensional system of active particles, the second equation in Eq. (89) in general form can be solved only numerically. However, in the two extreme cases examined above, this equation can be solved analytically. Namely, in the case of small values of the parameter $p_{0} \sqrt{\gamma / 2 D_{p}}=\sqrt{\tilde{g}^{2} / 2 \gamma D_{p}} \ll 1$ the solution of Eq. (89) is determined by

$$
\tilde{g} \approx-4 \pi^{2} \frac{\bar{g}}{\mathfrak{v}}\left(\frac{\pi \gamma}{2 D_{p}}\right)^{1 / 2},
$$




$$
p_{0} \approx 4 \pi^{2} \frac{\bar{g}}{\mathfrak{v} D_{p}}\left(\frac{\pi D_{p}}{2 \gamma}\right)^{1 / 2},
$$

and, as is easily seen directly, the ratio $p_{0} \sqrt{\gamma / 2 D_{p}}=\sqrt{\tilde{g}^{2} / 2 \gamma D_{p}} \ll 1$ can be reduced to the form Eq. (86), valid for the two-dimensional case.

At large values of the parameter $p_{0} \sqrt{\gamma / 2 D_{p}}=\sqrt{\tilde{g}^{2} / 2 \gamma D_{p}}, \quad p_{0} \sqrt{\gamma / 2 D_{p}}=$ $\sqrt{\tilde{g}^{2} / 2 \gamma D_{p}} \gg 1$ the solution of equation (89) is given by:

$$
\begin{aligned}
& \tilde{g} \approx-8 \frac{\pi \bar{g}}{\mathfrak{v}}\left(\frac{\pi \gamma}{2 D_{p}}\right)^{3 / 2} \frac{D_{p}}{\gamma}\left(1-2 \pi^{3} \frac{\bar{g}}{\mathfrak{v} D_{p}}\right)^{-1}, \\
& p_{0} \approx 4 \pi^{2} \frac{\bar{g}}{\mathfrak{v} D_{p}} \sqrt{\frac{\pi D_{p}}{2 \gamma}}\left|1-2 \pi^{3} \frac{\bar{g}}{\mathfrak{v} D_{p}}\right|^{-1} .
\end{aligned}
$$

Analysing Eq. (91) we find that at $\bar{g}>0$ in the domain of large values of the parameter the negative $\tilde{g}$ is only possible if $2 \pi^{3} \frac{\bar{g}}{\mathfrak{v} D_{p}}<1$. In this case the expression for $p_{0}$ can be simplified,

$$
p_{0} \approx \sqrt{\frac{2 D_{p}}{\pi \gamma}}\left|1-2 \pi^{3} \frac{\bar{g}}{\mathfrak{v} D_{p}}\right|^{-1},
$$

and the condition $\sqrt{\tilde{g}^{2} / 2 \gamma D_{p}} \gg 1$ can be replaced with an equivalent one,

$$
0<\frac{\bar{g}}{\mathfrak{v} D_{p}}<\frac{1}{2 \pi^{3}}
$$

Thus, we conclude that self-propulsion particles can be realized in the case of large values of the parameter $\sqrt{\tilde{g}^{2} / 2 \gamma D_{p}}$ and in three-dimensional system, but the criteria (94) in this motion differ significantly from that in the two-dimensional case, see Eq. (88).

It should be noted that if $\bar{g}=0$ the characteristic momentum $p_{0}$ is always zero, both in two- and three-dimensional cases, see Eq. (80) and the original equation (72). This should have been expected, since this case corresponds to the degeneration of the two bell-like peaks single-particle distribution function into a symmetrical one (with respect to $p=0$ ) of the Gaussian type, with parameters that coincide with those of Eqs. (70), (71).

It is necessary to emphasize once again that the example we considered in detail above is one of the special cases of the stationary solutions of equation (63). It is clear that the stationary states of the system, which satisfy the stationary variant of equation (63), can also have a more complex form, while remaining spatially homogeneous. We have chosen the type of solution (see (76) - (94)) precisely because it reproduces formulas that demonstrate the self-propulsion properties of the systems under consideration, which are similar to the self-propulsion results, for example, of Refs. [3, 18, 21]. It should be noted here that the self-propulsion behavior of active media in this paper is described as a collective effect and a consequence of the influence of spatially correlated noise. But at the same time, for the manifestation of the self-propulsion effect in the system, the particles (even if they are considered to be point) should have the property of head-tail asymmetry. That is, one of the reasons for the possible manifestation of the 
properties of the self-propulsion system are the individual characteristics of the particles - the structural units of the system. In our model, this was ensured by the dependence of the influence of noise on the magnitude and direction of the velocity of the particle itself. Thus, with the external similarity of the results of the present work and, for example, the work [18], the reasons leading to these results may be different. Recall that in [18] one particle is considered, in fact, which is logical for an approach that uses the Langevin equations as the initial equations for the individual motion of particles. The driving force arising in such a system is not a collective effect. However, it should still be borne in mind that a phenomenological approach was used in [18], where a nonlinear friction force in the medium was assumed and a very specific dependence of the friction coefficient on speed was postulated.

In concluding the section, it is also necessary to reiterate the following circumstance. Above in a number of places, we specifically separated the cases of two-dimensional and three-dimensional systems. From the procedure for constructing the kinetic theory of active media in the microscopic approach described in the paper, it can be seen that there is no conceptual difference between the derivation of equations in two- and three-dimensional cases. However, the situation looks this way only after the equations of motion (6) - (9) are written out. These equations are formulated to replace the two-dimensional Langevin equations, which usually underlie the construction of the kinetic theory of active media. The generalization of the initial equations of motion for structural units, as could be seen, is completely non-trivial. In fact, as was noted in the Introduction, historically, the situation has developed in such a way that, in the earlier works of other authors, when constructing the kinetic theory of active media, as a rule, they proceeded from two-dimensional Langevin type equations (10). In some cases, the interactions between the structural units of the system were included in the above-mentioned Langevin equations, see, for example, [1, 2, 3, 4, 5, 6, 19, 20, 21]. However, building a consistent microscopic approach to deriving the kinetic equations of such media requires going beyond the scope of the Langevin equations as the initial equations of motion for the structural units of the system. Instead of the Langevin equations, it is necessary to formulate the generalized Hamilton equations, that is, to base the first principles of classical theoretical mechanics. For this reason, in this paper we proceeded from the generalized Hamilton equations, which allow both naturally to include interactions between particles (including dissipative) and a three-dimensional view of the stochastic effect on the system, depending on speed and coordinates of structural units. And in order to compare the results that follow from our theory in certain particular cases, with the results of other authors (for two-dimensional systems), we also separated in some cases the results for three-dimensional and two-dimensional systems. It should also be noted that in the framework of the model we are considering, the conditions (or criteria) for the existence of self-propulsion in the system may differ for two-dimensional and three-dimensional systems (see, for example, (88) and (94). At the same time, the very possibility of the existence of such self-propulsion modes in both the two-dimensional and three-dimensional cases is not prohibited. 


\section{Conclusion}

In this paper we propose a microscopic approach to the construction of the kinetic theory of many-particle systems with dissipative and potential interactions in the presence of active fluctuations. The approach is based on a generalization of BogolyubovPeletminskii reduced description method applied to the systems of many active particles. The microscopic approach allows us to construct the kinetic theory of active particles in the case of two-dimensional and three-dimensional systems as well, in presence of a non-linear friction and local nature of active fluctuations. We obtain general kinetic equations for these systems in the case of a weak interaction between the particles (both potential and dissipative) and low-intensity active fluctuations. We define some particular cases in which the derived kinetic equations have solutions that match with the results for the systems of active particles known from earlier works by other authors. It is also shown that one of the consequences of the local nature of the active fluctuations is a manifestation of a head-tail asymmetry and a self-propulsion, typical for systems of active particles, even in the case of a linear friction, see Eqs. (83) - (94).

We remind in this context that formulas (83) - (94) describe only two special limiting cases of two-dimensional and three-dimensional systems with the self-propulsion particles. The expressions obtained for a two-dimensional case coincide with those derived in $[3,21]$. However, in our approach the nature of the phenomenon of selfpropulsion is associated with a local (individual) exposure to the particles of the external stochastic field with active fluctuations, see Eq. (9). Besides, the parameters of the selfpropulsion motion are self-consistently expressed by the internal characteristics of manybody system, namely the number density of particles in the system, the parameters of the dissipative function, and characteristics of the external influence, that is the pair correlation function of the active random field. Note that the stationary direction of the head-tail asymmetry within the spatially homogeneous model (see Eqs. (65), (66)) can not be determined. To define it, we should introduce an interaction, even an arbitrarily small, but violating the spatial isotropy of the problem. In this sense, the situation expressed by Eqs. (79) - (88), is similar to the situation with a phase transition to the magnetic ordering in ferromagnets, see, e.g., [42]. As it is known, the value of the total magnetic momentum in a ferromagnet in the main approximation is determined by the isotropic exchange interaction. The direction of the magnetization is at the same time given by non-isotropic weak relativistic interactions. The situation with the appearance of anisotropy in the momentum space as a result of a phase transition in a normal Fermi-liquid with a weakly anisotropic interaction was also considered in [43].

At that point we note that the kinetic equations (55) - (57) are general in the sense that they describe quite a number of different many-particle systems, both two-dimensional and three-dimensional ones, with active local fluctuations, space homogeneous and inhomogeneous as well, including different variations of non-linear friction. The study of various particular cases of solutions of kinetic equations (55) - (57) is beyond the scope of this paper. As outlined above, the main objective of 
this work is the development of microscopic approach to the derivation of the general kinetic equations for active particles with non-linear friction under the influence of active fluctuations, including a generalization to the case of the three-dimensional systems.manuscript

We also note that the microscopic approach suggested here for the construction of the kinetic theory of many-particle systems with dissipative interaction and active fluctuations allows for further generalization, e.g., to the case of simultaneous presence of both active and passive fluctuations. Furthermore, a non-Gaussian stochastic nature of external effects can be taken into account, see also in this regard [18].

\section{Acknowledgements}

The authors would like to thank Prof. A. Chechkin for fruitful discussions and valuable comments on the paper.

OS acknowledges the supported by the Spanish Ministry of Economy and Competitiveness MINECO through BCAM Severo Ochoa excellence accreditation SEV2013-0323 and SEV-2017-0718.

\section{References}

[1] Ramaswamy S 2010 Annu. Rev. Condens. Matter Phys. 1 323-345

[2] Vicsek T and Zafeiris A 2012 Physics Reports 517 71-140

[3] Romanczuk P, Bär M, Ebeling W, Lindner B and Schimansky-Geier L 2012 The European Physical Journal Special Topics 202 1-162

[4] Marchetti M, Joanny J, Ramaswamy S, Liverpool T, Prost J, Rao M and Simha R A 2013 Reviews of Modern Physics $\mathbf{8 5} 1143$

[5] Pismen L, Kruse K and (Eds) 2014 Eur. Phys. J. Spec. Top. 223

[6] Hauser M and Schimansky-Geier L 2015 Eur. Phys. J. Spec. Top. 224 1147-1150

[7] Abaid N, Butail S, Porfiri M and Spinello D 2015 Eur. Phys. J. Spec. Top. 224 3109-3117

[8] Skjeltorp A, Helgesen G and (Eds) 2016 Eur. Phys. J. Spec. Top. 225

[9] Couzin I D, Levin S A and (Eds) 2015 J. Stat. Phys. 158

[10] Bertin E, Droz M and Grégoire G 2006 Physical Review E 74022101

[11] Bertin E, Droz M and Grégoire G 2009 Journal of Physics A: Mathematical and Theoretical 42 445001

[12] Bertin E, Baskaran A, Chaté H and Marchetti M C 2015 Physical Review E 92042141

[13] Ihle T 2011 Physical Review E 83030901

[14] Ihle T 2014 The European Physical Journal Special Topics 223 1293-1314

[15] Ihle T 2013 Physical Review E 88040303

[16] Chou Y L, Wolfe R and Ihle T 2012 Physical Review E 86021120

[17] Romensky M, Lobaskin V and Ihle T 2014 Physical Review E 90063315

[18] Romanczuk P and Schimansky-Geier L 2011 Physical review letters 106230601

[19] Grossmann R, Schimansky-Geier L and Romanczuk P 2012 New Journal of Physics 14073033

[20] Grossmann R, Schimansky-Geier L and Romanczuk P 2013 New Journal of Physics 15085014

[21] Lobaskin V and Romenskyy M 2013 Physical Review E 87052135

[22] Bogoliubov N 1959 Providence College, Providence, Rhode Island

[23] Akhiezer A I and Peletminskii S V 1981 Methods of statistical physics (Pergamon)

[24] Prigogine I 1962 Non-equilibrium statistical mechanics (Interscience Publisher) 
[25] Zubarev D 1974 Nonequilibrium Statistical Thermodynamics (Springer)

[26] Klimontovich Y 1986 Statistical Physics (New York: Harwood Academic Publishers)

[27] Zubarev D, Morozov V and Roepke G 1996 Statistical Mechanics of Nonequilibrium Processes (Akademy, Berlin)

[28] Luzzi R, Vasconcellos Á R and Ramos J G 2002 Predictive Statistical Mechanics: A Nonequilibrium Ensemble Formalism vol 122 (Springer Science \& Business Media)

[29] Peletminskii S, Slyusarenko Y V and Sokolovsky A 2003 Physica A: Statistical Mechanics and its Applications 326 412-429

[30] Sliusarenko O Y, Chechkin A and Slyusarenko Y V 2015 Journal of Mathematical Physics 56 043302

[31] Goldhirsch I, Peletminskii A, Peletminskii S and Sokolovsky A 2013 arXiv preprint arXiv:1307.3466

[32] Landau L D, Lifshits E M and Pitaevskiǔ L P 1980 Statistical physics vol 5 (Pergamon)

[33] Laskin N, Peletminskii S and Prikhod'ko V 1998 Journal of Physical Studies 2 6-15

[34] Furutsu K and Novikov E 1963 J. Res. N.B.S. D-67 303

[35] Novikov E 1964 Zh. Eksp. Teor. Fiz. 471919

[36] Moiseev S, Tur A and Yanovsky V 1976 Zh. Eksp. Teor. Fiz. 711062

[37] Astumian R D and Hänggi P 2002 Phys. Today 5533

[38] Reimann P 2002 Physics reports 361 57-265

[39] Weinstock J 1963 Phys. Rev. 139470

[40] Kawasaki K and Oppenheim I 1965 Physical Review 139 A1763

[41] Uhlenbeck G E and Ford G W 1963 Lectures in statistical mechanics (Providence Rhode Island)

[42] Akhiezer A I, Bar'yakhtar V G and Peletminskii S V 1968 Spin waves (North-Holland Publishing Company Amsterdam)

[43] Peletminsky A, Peletminsky S and Slyusarenko Y V 1999 Low Temperature Physics 25 153-160 